Pontifícia Universidade C Católica

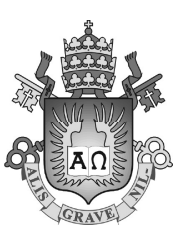

Rafael de Pinho André

\title{
Using Body Sensor Networks and Human Activity Recognition Classifiers to Enhance the Assessment of Form and Execution Quality in Functional Training
}

Thesis presented to the Programa de Pós-graduação em Informática of PUC-Rio in partial fulfillment of the requirements for the degree of Doutor em Informática.

Advisor: Prof. Alberto Barbosa Raposo 

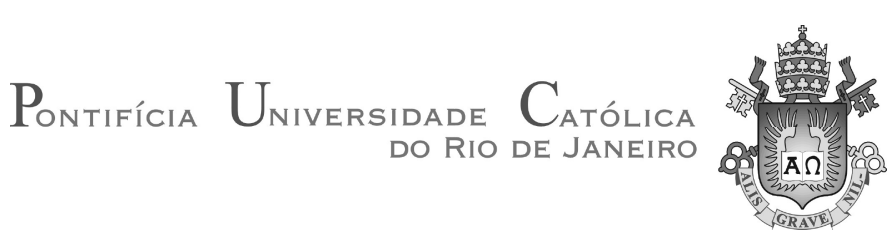

Rafael de Pinho André

\begin{abstract}
Using Body Sensor Networks and Human Activity Recognition Classifiers to Enhance the Assessment of Form and Execution Quality in Functional Training
\end{abstract}

\begin{abstract}
Thesis presented to the Programa de Pós-graduação em Informática of PUC-Rio in partial fulfillment of the requirements for the degree of Doutor em Informática. Approved by the Examination Committee.
\end{abstract}

\author{
Prof. Alberto Barbosa Raposo \\ Advisor \\ Departamento de Informática - PUC-Rio \\ Prof. Hugo Fuks \\ Pontifícia Universidade Católica do Rio de Janeiro - PUC-Rio \\ Profa. Simone Diniz Junqueira Barbosa \\ Pontifícia Universidade Católica do Rio de Janeiro - PUC-Rio \\ Prof. André Soares Monat \\ Universidade do Estado do Rio de Janeiro - UERJ \\ Profa. Denise Del Re Filippo \\ Universidade do Estado do Rio de Janeiro - UERJ
}


All rights reserved.

\section{Rafael de Pinho André}

Bachelor's degree in computer engineering and Master's degree in Computer Science by the Pontifícia Universidade Católica do Rio de Janeiro. MBA in IT management by the same university. Worked as a software engineer and consultant for over a decade, and now is responsible for the IT management and governance of the regional labour court.

Bibliographic data

André, Rafael de Pinho

Using body sensor networks and human activity recognition classifiers to enhance the assessment of form and execution quality in functional training / Rafael de Pinho André; advisor: Alberto Barbosa Raposo. - 2020.

70 f. : il. color. ; $30 \mathrm{~cm}$

Tese (doutorado)-Pontifícia Universidade Católica do Rio de Janeiro, Departamento de Informática, 2020.

Inclui bibliografia

1. Informática - Teses. 2. Reconhecimento de atividade humana. 3. Saúde e ergonomia. 4. Educação física. 5. Dispostivos loT (Internet das Coisas). 6. Computação vestível e sensoriamento. I. Raposo, Alberto Barbosa. II. Pontifícia Universidade Católica do Rio de Janeiro. Departamento de Informática. III. Título. 
To my parents, for their support and encouragement throughout my life. 


\section{Acknowledgments}

I would like to first thank my advisor for his guidance and understanding, and specially for all the help provided in many moments of crisis. This work would no be possible without his support.

Then I wish to sincerely thank my former advisor, Hugo Fuks, who taught me how to conduct research and understand the academic world.

This study was financed in part by the Coordenação de Aperfeiçoamento de Pessoal de Nível Superior - Brasil (CAPES) Finance Code 001

Finally I wish to thank my late colleague Marcio Cunha, for all his technical

guidance and academic advice, key to the development of the first prototype and the publication of my first doctoral research paper. 


\section{Abstract}

André, Rafael de Pinho; Raposo, Alberto (Advisor). Using Body Sensor Networks and Human Activity Recognition Classifiers to Enhance the Assessment of Form and Execution Quality in Functional Training. Rio de Janeiro, 2020. 70p. Tese de doutorado - Departamento de Informática, Pontifícia Universidade Católica do Rio de Janeiro.

Foot and knee pain have been associated with numerous orthopedic pathologies and injuries of the lower limbs. From street running to CrossFit ${ }^{\mathrm{TM}}$ functional training, these common pains and injuries correlate highly with unevenly distributed plantar pressure and knee positioning during long-term physical practice and can lead to severe orthopedic injuries if the movement pattern is not amended. Therefore, the monitoring of foot plantar pressure distribution and the spatial and temporal characteristics of foot and knee positioning abnormalities is of utmost importance for injury prevention. This work proposes a platform, composed of an IoT wearable body sensor network and a Human Activity Recognition (HAR), to provide realtime feedback of functional exercises, aiming to enhance physical educators capability to mitigate the probability of injuries during training. We conducted an experiment with 12 diverse volunteers to build a HAR classifier that achieved about $87 \%$ overall classification accuracy, and a second experiment to validate our physical evaluation model. Finally, we performed a semi-structured interview to evaluate usability and user experience issues regarding the proposed platform.

Aiming at a replicable research, we provide full hardware information, system source code and a public domain dataset.

\section{Keywords}

Human Activity Recognition; Health and Ergonomics; Physical education; IoT devices; Wearable Computing and Sensing; Mobile sensing applications. 


\section{Resumo}

André, Rafael de Pinho; Raposo, Alberto. Utilizando Redes de Sensores Corporais e Classificadores de Reconhecimento de Atividade Humana para Aprimorar a Avaliação de Qualidade de Forma e Execução em Treinamentos Funcionais. Rio de Janeiro, 2020. 70p. Tese de Doutorado - Departamento de Informática, Pontifícia Universidade Católica do Rio de Janeiro.

Dores no pé e joelho estão relacionadas com patologias ortopédicas e lesões nos membros inferiores. Desde a corrida de rua até o treinamento funcional CrossFit ${ }^{\mathrm{TM}}$, estas dores e lesões estão correlacionadas com a distribuição iregular da pressão plantar e o posicionamento inadequado do joelho durante a prática física de longo prazo, e podem levar a lesões ortopédicas graves se o padrão de movimento não for corrigido. Portanto, o monitoramento da distribuição da pressão plantar do pé e das características espaciais e temporais das irregularidades no posicionamento dos pés e joelhos são de extrema importância para a prevenção de lesões. Este trabalho propõe uma plataforma, composta de uma rede de sensores vestíveis e um classificador de Reconhecimento de Atividade Humana (HAR), para fornecer feedback em tempo real de exercícios funcionais, visando auxiliar educadores físicos a reduzir a probabilidade de lesões durante o treinamento. Realizamos um experimento com 12 voluntários diversos para construir um classificador HAR com aproximadamente de $87 \%$ de precisão geral na classificação, e um segundo experimento para validar nosso modelo de avaliação física. Por fim, realizamos uma entrevista semiestruturada para avaliar questões de usabilidade e experiência do usuário da plataforma proposta.

Visando uma pesquisa replicável, fornecemos informações completas sobre o hardware e o código fonte do sistema, e disponibilizamos o conjunto de dados do experimento.

\section{Palavras-chave}

Reconhecimento de Atividade Humana; Saúde e Ergonomia; Educação Física; Dispostivos IoT; Computação Vestível e Sensoriamento; Aplicações de Sensores Móveis 


\section{Table of contents}

1 Introduction 13

2 Literature Review $\quad 20$

2.1 User Feedback and Performance Assessment 22

2.2 Activity Recognition 23

2.3 Prevention and Treatment of Injuries and Diseases 25

2.4 Literature Review Discussion 26

3 IoT Device Prototype $\quad 30$

3.1 Analysis of the Sensor Mix 33

4 First Experiment and HAR Classifier Building 38

4.1 Planning the Experiment 38

4.2 Conducting the Experiment 45

4.3 Data Acquisition 48

4.4 Data Processing, Feature Extraction and Selection 49

4.5 Classification and Validation 50

$5 \quad$ Second Experiment and Methodology Evaluation 52

5.1 The Second Experiment 52

5.1.1 The Interview

$\begin{array}{lll}6 & \text { Conclusion } & 58\end{array}$ 


\section{List of figures}

Figure 1.1 Obesity prevalence in Brazil. Ministério da Saúde. 14

Figure 2.1 Recent surge of interest in investigating the use of $\begin{array}{ll}\text { embedded sensors in shoes and insoles. } & 21\end{array}$

$\begin{array}{lll}\text { Figure 3.1 GCF sensor positioning. } & 31\end{array}$

Figure 3.2 The Particle.io Electron. 32

Figure 3.3 The LSM9DS1 IMU. 33

Figure 3.4 The MPL3115A2 barometer. 34

Figure 3.5 The RFD77402 distance sensor. 35

Figure 3.6 Shoe external protective case. 35

$\begin{array}{lll}\text { Figure } 3.7 & \text { Knee external protective case. } & 36\end{array}$

Figure 4.1 Hip Extensors: main and auxiliary muscles [90]. 39

Figure 4.2 Basic squat: start and end positions [26]. 40

Figure 4.3 Sumo squat: start and end positions [26]. 41

Figure 4.4 Front lunge: start and end positions [26]. 42

Figure 4.5 Side lunge: start and end positions [26]. 42

Figure 4.6 Step-up: movement pattern [26]. 43

Figure 4.7 Difference between full and partial extensions [78]. 43

Figure 4.8 Angles of hip drive movement patterns [78]. 44

Figure 4.9 Difference between the correct back positioning, on the

left, and lumbar overextension, on the right [78]. 45

Figure 4.10 Mid-foot balance point [78]. 46

Figure 4.11 Unbalance due to mid-foot positioning mistakes [78]. $\quad 48$

Figure 4.12 Correct heel positioning and feet angle [78]. 48 


\section{List of tables}

$\begin{array}{lll}\text { Table 1.1 Weisenthal et al. [97] } & 17\end{array}$

Table 2.1 Literature review metadata: methods and results. 27

Table 2.2 Literature review metadata: sensor positioning. 29

Table 3.1 Comparison between US men's sizes and other standards. 31

Table 3.2 Single sensor classifier accuracy. 36

$\begin{array}{lll}\text { Table 3.3 Sensor Fusion classifier accuracy. } & 37\end{array}$

Table 4.1 Main physical characteristics of each participant 47

Table 4.2 Data collection experiment exercise routines. 47

Table 4.3 Results per participant $\quad 51$

Table 5.1 Main physical characteristics of each participant 52

Table 5.2 Model validation experiment exercise routines. 53

Table 5.3 Assessment model evaluation $1 \quad 54$

Table 5.4 Assessment model evaluation 2 


\section{List of Abreviations}

ADC - Analog to Digital Converter

ADHD - Attention Deficit Hyperactivity Disorder

ADL - Activities of Daily Living

BMI - Body Mass Index

BSN - Body Sensor Network

EAI - Exercise Addiction Inventory

FPB - Foot Plantar-Based

FSR - Force Sensitive Resistor

GCF - Ground Contact Force

HAR - Human Activity Recognition

HIFT - High Intensity Functional Training

IBGE - Instituto Brasileiro de Geografia e Estatística

IMU - Inertial Motion Unit

IoT - Internet of Things

JSON - JavaScript Object Notation

LED - Light-Emitting Diode

mAh - Milliampere Hour

PESS - Physical Education and School Sport

PHA - Patient Handling Activities

PNAD - Pesquisa Nacional por Amostra de Domicílios

PTF - Polymer Thick Film

RIS - Research Information Systems

RSI - Repetitive Strain Injuries

ToF - Time of Flight

VCSEL - Vertical Cavity Surface Emitting Laser

WoD - Workout of the Day 
And you, my father, there on the sad height, Curse, bless, me now with your tears, I pray. Do not go gentle into that good night. Rage, rage against the dying of the light.

Dylan Thomas, In Country Sleep And Other Poems. 


\section{1 Introduction}

Physical education and school sport (PESS) aims at (i) developing cognitive capacity and motor skills, (ii) teaching about health and the benefits of physical activity and (iii) fostering emotional intelligence [48]. Recent research on successful PESS programs shows a strong correlation between physical activity, learning performance and academic success [77]. Many studies, such as [11] and [12], identify that an adequate exposure to PESS increases energy expenditure, allowing for the maintenance of a healthy weight and reducing chances of experiencing chronic disease factors and becoming obese. However, although adequate exposure to PESS is considered to be between 30 and 60 minutes per day [86], studies such as [93] and [65] point out that the average physical activity level of children and young adults worldwide is low and decreasing, and there is a correlated increase in obesity and chronic disease factors.

Obesity is a largely preventable chronic disorder, with genetic, behavioral, socioeconomic and environmental origins, classified officially as a disease (ICD-10 E66.0) [43]. It is defined as an abnormal accumulation of body fat that has an adverse effect on health, leading to significant long-term health consequences, such as the development of musculoskeletal disabilities, debilitating morbidity, depression, type 2 diabetes, coronary heart disease, osteoarthritis and the increase of the risk of developing certain cancers and influencing their outcomes [1]. The current most widely used criteria for classifying obesity is the body mass index (BMI). Obesity has become a rapidly growing public health problem, predominantly by cause of (i) transition to processed foods and high calorie diets, (ii) increasingly sedentary lifestyle with reduced levels of exercise, (iii) falling number of hours slept, and (iv) changes in the built environment, such as urbanization, industrialization, and mechanized transport. As discussed in [43], many countries have witnessed the prevalence of obesity in its citizens double, and even quadruple, in recent years. Now, the growth trends in overall obesity in most developed countries leveled off, although morbid obesity continues to climb, including among children - which suggests a burden to healthcare systems in the next decades. In addition, obesity prevalence in most developing countries continues to trend upwards toward US levels - the 
country with higher obesity rate [69]. The recent increase in the prevalence of obesity in Brazil is shown in Figure 1.1.

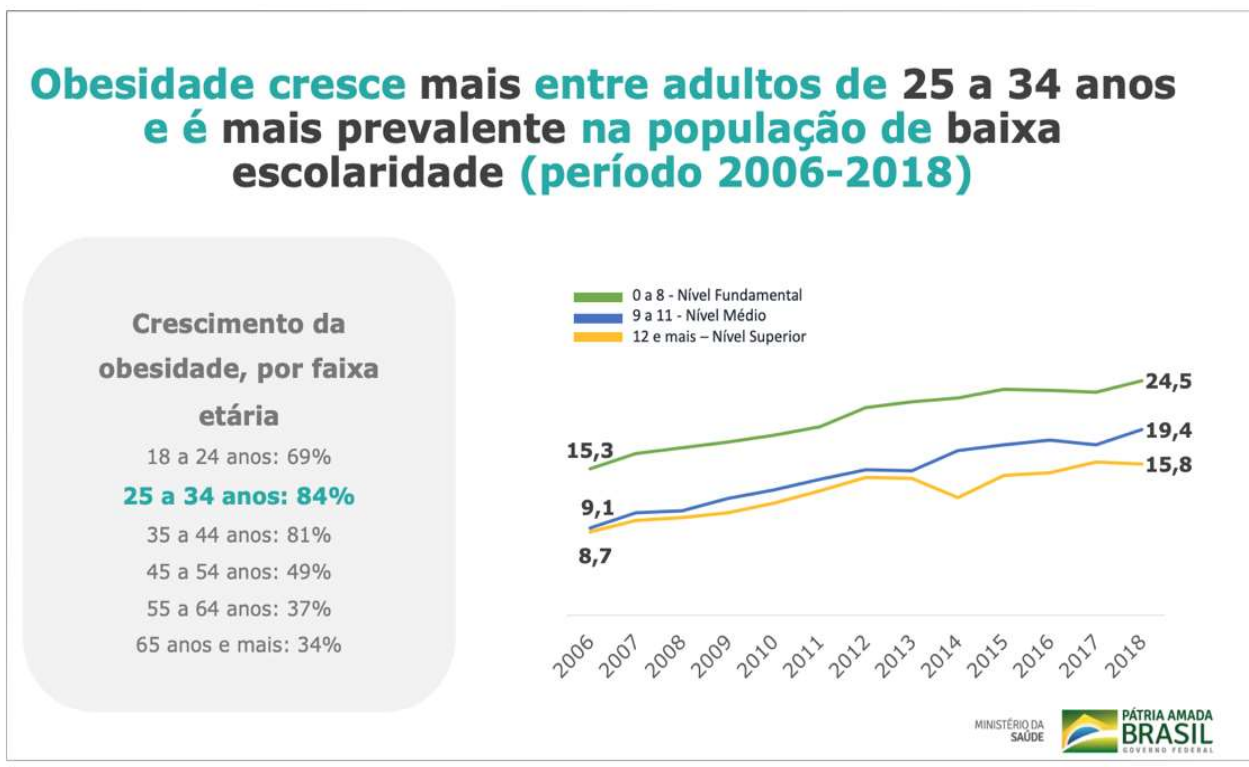

Figure 1.1: Obesity prevalence in Brazil. Ministério da Saúde.

The epidemic of overweight and obesity, along with the worldwide decrease of physical activity levels and the prevalence of musculoskeletal pain, presents, then, a major challenge to physical educators around the world. Therefore, the assembling of physical education programs that are compelling and safe to individuals in diverse groups has seen an intense increase in research and investigation. Over the last 10 years, functional training exercises became a popular method to improve muscular and cardiovascular fitness, and many studies, such as [10] and [32], point out the benefits of this type of training regimen - while also suggesting that functional training practitioners have increased risk of foot, knee and lower back pain and related injuries. Nevertheless, works such as [96] and [66] point out a twofold increase of the prevalence of knee pain injuries, whereas works such as [25] show a threefold increase of the prevalence of lower back pain and injuries. The prevalence of lower back pain in Brazil is 13.5\% [44], whilst the prevalence of knee pain is $8 \%[91]$.

Foot and knee pain have been associated with numerous orthopedic pathologies and injuries of the lower limbs. From high intensity functional training (HIFT), such as CrossFit ${ }^{\mathrm{TM}}$, to Extreme Conditioning Programs, such as those employed by the military and competing athletes, lower limb injuries correlate highly with unevenly distributed plantar pressure and knee posi- 
tioning during long-term physical practice and can lead to severe orthopedic injuries if the movement pattern is not amended. Therefore, the monitoring of foot plantar pressure distribution and the spatial and temporal characteristics of foot and knee positioning abnormalities is of utmost importance for Repetitive Strain Injury (RSI) prevention. The most common methods employed by physical educators for the diagnosis of plantar pressure and knee positioning abnormalities are based on human observational approach, predominantly subjective, which is neither reliable nor scalable to a large group of individuals.

CrossFit ${ }^{\mathrm{TM}} \mathrm{HIFT}$ is a strength and conditioning program promoted as both a lifestyle philosophy and a competitive sport. It incorporates a wide range of exercises, typically performed in a timed or circuit format, including routines adapted from (i) Olympic weightlifting and power-lifting, (ii) gymnastics, and (iii) traditional aerobic exercises, such as running and rowing [64]. CrossFit" is described by its proponent as a "constantly varied, highintensity, functional movement workout" [30]. Formally established in 2000, after two decades there are over 12,000 certified and registered CrossFit fitness centers worldwide - around 450 certified and registered CrossFit fitness centers in Brazil, with approximately 40,000 Athletes. Although the majority of the CrossFit ${ }^{\mathrm{TM}}$ Athletes are above 21 years of age worldwide, there has been studies, such as [20], suggesting that CrossFit ${ }^{\mathrm{TM}}$ should be incorporated into the physical education curriculum of secondary schools. These studies do not address the long-term health risks of HIFT practice during adolescence. A typical CrossFit ${ }^{\mathrm{TM}}$ class is an hour long and includes (i) a warm-up, (ii) a skill development segment, (iii) the high-intensity Workout of the Day (WOD), and (iv) a period of individual or group stretching.

Many studies focused on CrossFit ${ }^{\mathrm{TM}}$, such as [9] and [85], indicate that this combination of high intensity exercise performed in a group-based setting is effective in improving general fitness, aerobic capacity, muscular endurance and body composition. The same studies also suggest that highly technical exercises performed for high numbers of repetitions, with the subject under the influence of fatigue, leads to poor technical execution and thus pose an RSI risk to participants. In addition to that, a joint paper presented by the Consortium for Health and Military Performance and the American College of Sports Medicine expresses concern over the high injury rates of extreme conditioning programs and correlates these injuries with the development of rhabdomyolysis, a serious syndrome that can lead to renal failure [33].

In addition to the risks of physical injuries, some studies, such as [51], investigate the prevalence of exercise addiction in CrossFit ${ }^{\mathrm{TM}}$ populations, employing the Exercise Addiction Inventory (EAI). The EAI is a self-report 
that examines an individual's beliefs toward exercise, proposed in [92] and used in some of the surveyed works. The inventory is composed of six statements, concerning: (i) the importance of exercise to the individual, (ii) relationship conflicts due to exercise, (iii) how mood changes with exercise, (iv) the amount of time spent exercising, (v) the outcome of missing a workout, and (vi) the effects of decreasing physical activity. Athletes addicted to physical practice are at a greater risk of negative consequences related to their exercise addiction, such as injuries, illness, loss of social relations and associated mental disorders [51]. The study found that there was no significant difference between CrossFit ${ }^{\mathrm{TM}}$ and other fitness activities, although the addiction is more prevalent in young practitioners - below 30 years of age - and in males.

As discussed in [97], the injury rates among CrossFit ${ }^{\mathrm{TM}}$ practitioners vary according to the type of training program followed: (i) gymnastics-based exercises, (ii) powerlifting-based exercises, or (iii) endurance-based exercises. In gymnastics-based exercise programs, the shoulder is the most commonly injured body area. In powerlifting oriented exercise programs, the most commonly injured body area is the lower back. Lastly, in endurance-based exercise programs the most commonly injured body areas, in order of occurrence, are the shoulder, the lower back and the knee. This study also suggested that there was no significant difference in injury rate based on age, length of training or weights lifted by the participants. However, males were significantly more liked to injure themselves than females. Although the overall injury rate found in this study was around $20 \%$ - injury rate varied according to the type of training program followed and the methodology employed in the survey -, all participants were professional athletes. Another research [36], with a broader participant base composed mostly of non-professionals, found an injury rate of $73.5 \%$ considering the six months prior to the evaluation, and $10.5 \%$ of those that sustained injuries required surgical intervention. Many studies, such as [97] and [17], showed a significant correlation between injury level and coach supervision, as seen on Table 1.1. No study investigated the correlation between injure rates and coach experience, feedback quality or class sizes. The CrossFit ${ }^{\mathrm{TM}}$ organization does not directly supervise its certified and registered CrossFit ${ }^{\mathrm{TM}}$ fitness centers, nor does it require them to perform the programming that is produced each day by CrossFit ${ }^{\text {TM }}$ headquarters in Washington, DC. Certified and registered CrossFit ${ }^{\mathrm{TM}}$ fitness center owners are required to have a CrossFit ${ }^{\mathrm{TM}} \mathrm{L} 1$ certification, yet no further training is necessary. Therefore, there is a wide variation in instruction and feedback quality between CrossFit $^{\mathrm{TM}}$ Certified and registered fitness centers based on the experience and personal characteristics of the owner and coaches [97]. Enhancing the ca- 


\begin{tabular}{lll}
$\begin{array}{l}\text { Injury Rate Versus Trainer Presence } \\
\text { Trainer involvement }\end{array}$ & No Injury & Injury \\
\hline All the time & $85.4 \%$ & $14.6 \%$ \\
Most of the time & $75.6 \%$ & $24.4 \%$ \\
Some of the time & $69.0 \%$ & $31.0 \%$
\end{tabular}

Table 1.1: Weisenthal et al. [97]

pability of a trainer to provide adequate feedback to CrossFit ${ }^{\mathrm{TM}}$ practitioners is, then, key to mitigate the probability of RSI by cause of training.

Human Activity Recognition (HAR) is an active and fast growing field of research, and its methods using feet and knee movement and posture information are recently drawing attention of different research fields such as mobile healthcare, sports performance and ergonomics. Researchers investigate the recognition of human movement patterns and behaviors to better understand our actions and their context, to detect misguided action and to help people perform better in their daily life or professional activities. Traditionally, the equipment used to track and process these movements patterns were invasive, expensive and unsuited for outdoor experiments, but the development of Internet of Things (IoT) wearable technologies allowed researchers to investigate HAR related questions without the constraints of a laboratory or a prepared environment. From fall risk assessment of elderly patients [4] and rehabilitation of stroke patients [21] to energy expenditure estimation of Activities of Daily Living (ADL) $[80,81]$ and the recognition of a performer's dancing steps to provide real-time feedback [94], literature shows promising results and interesting applications of IoT technologies. Currently, there are two main approaches for collecting user's feet movement and posture data: (i) image processing and (ii) wearable sensors. Image processing techniques require camera installation, thus making outdoor operation more expensive and rising privacy concerns [16]. By contrast, the wearable sensors approach used by our work allows for easier outdoor operation, trading-off lightning and image concerns for issues regarding skin allergies, battery charges, prototype resilience and the positioning of sensors.

This work presents a study for foot and knee movement and posture analysis of individuals to help the assessment and correction of their movements during training, aiming to mitigate the probability of RSI during the execution of functional exercises. Our solution employs an IoT wearable device with a novel sensor array and a machine learning HAR activity classifier for movement pattern recognition. The wearable device comprises three components: an US men's size 8.5 insole that houses the plantar pressure sensors, an 
external protective case that houses the microcontroller and the foot sensors and a knee band that houses the knee sensors. It is equipped with a set of 16 sensors and can collect detailed foot and knee movement and posture information every $10 \mathrm{~ms}$, providing a feature-rich stream of data for processing and analysis. The machine learning HAR activity classifier employs a Random Forest algorithm to recognize, within a set of 13 activities, whether the movement pattern is correct or inaccurate. The experimental results show that the HAR classifier achieves an overall accuracy of $87.08 \%$ for the recognition of activities and $67.51 \%$ for the user feedback. After classification, the IoT wearable device uses a component that provides real-time haptic feedback to the user to warn whenever an inaccurate movement pattern is performed.

We performed a literature review on HAR using Internet of Things (IoT) devices and wearable sensors, discussed in chapter 2 , which unfolded the intense growth on the number of publications related to the analysis of feet posture and movement in recent years. Throughout the review, we observed that (i) all works aimed at a complete substitution of the human agents, (ii) most works employed a small number of participants, usually 5 or less, (iii) the number or activities recognized - i.e the size of the assessed activity model - is often small, with a median of 3 and a mode of 1, (iv) there was no diversity of participants' profiles, (iv) no previous work thoroughly addressed the relevance of the sensors employed for the assessed activity model, (v) few works provided public datasets for benchmarking, (vi) there is no sufficient information on sensors deployment, (vii) few works addressed their sample rates and time window size choices and (viii) few works addressed the positioning of the plantar pressure sensors. The lack of published datasets, the insufficient information for the replication of these studies and the overall obliviousness of other work's results points to a lack of maturity of the area.

Two experiments were conducted to develop and validate the functional exercise execution quality assessment model. The objective of the first experiment was to build a machine learning HAR activity classifier, and it employed twelve volunteers carefully selected for their diverse characteristics, since a common limitation of the surveyed works is the prevalence of homogeneous participants. We employed participants from both genders and diverse age categories, some of which with disabilities, such as class II obesity, severe knee injury and blindness. Each participant performed 3 sessions of 30 minutes of functional training monitored by a certified physical educator, following a routine consisting of the 13 activities of our activity model. Subsequently, we conducted a second experiment with nine of the original twelve participants, consisting of a 30-minute session followed by a semi-structured interview to 
evaluate usability and user experience questions. The objective of the second experiment was to validate our HAR classifier and the quality assessment model. Our results demonstrate the feasibility of our real-time feedback system and the supporting role it can perform for early intervention and prevention of orthopedic injuries, by providing a mobile, easy to use and affordable long-term monitoring of individuals.

The main contributions of this work are an IoT device blueprint for HAR research with a novel sensor array, a public domain dataset obtained from an experiment with 13 diverse individuals and, above all, a method for enhancing the capability of health professionals to assess the quality of execution of functional exercises. In addition to that, this work also presents (i) a comprehensive literature review of wearable-based HAR research using feet movement and posture information, (ii) an auxiliary literature review focused on HAR research using wearable sensors for collecting feet movement and posture information and (iii) an analysis of the relevance of different types of sensors for the building of a HAR classifier based on feet movement and posture information.

The main and auxiliary literature reviews are presented in chapter 2 . In chapter 3 we detail the design process of the IoT device prototype and the sensor deployment choices, in addition to hardware and software replication information. The experiment conducted to collect activity data, and its details, is discussed in chapter 4 , along with the development of the activity model and the classifier. In chapter 5 we describe the second experiment, in which the proposed model of quality assessment for functional exercises is validated, and the usability and user experience evaluation, addressing the main concerns of the prototype usage and the quality assessment process. Conclusion and future work are discussed in chapter 6. 


\section{2 \\ Literature Review}

This chapter presents a literature review about wearable-based research projects using feet and knee movement and posture information focused on health and sports activities. We conducted the literature review in four steps: (i) definition of a research question and its sub questions, (ii) formulation of a search query string, (iii) definition of exclusion criteria and (iv) completion of a quantitative and qualitative data analysis. The research question posed in this work is: What are the wearable-based HAR research projects conducted in recognition of sports and health-related activities using feet movement and posture information?

This research question was broken down into six sub-questions:

- RQ1. How was the incorrect execution of activities addressed?

- RQ2. How was the activity model structured?

- RQ3. How was the model validated?

- RQ4. What activity classifier was built?

- RQ5. How much data was analysed?

- RQ6. What hardware and software setting - types, quantities and locations of the sensors - were assembled?

Based on these questions, we formulated the following a search query string: ((((Daily Activities) OR Activity Recognition) OR Monitoring) OR Detection) AND (((((Insole) OR Foot) OR Knee) OR Plantar) OR Shoe) OR Gait)). Results were refined by the publication year range to exclude works not published in the last 12 years, obtaining over four hundred search results combining the ACM Digital Library, IEEE Xplore, SciELO and Springer databases. The publication year range criteria was employed to exclude works based on deprecated hardware or software components. Our search suggests a recent surge of interest in investigating the use of embedded sensors in shoes and insoles for gait and ADL monitoring and analysis, as shown in Figure 2.1. We defined a strict exclusion criteria, as follows:

- Image processing HAR or the use of other non-wearable sensors; 
- Research about plantar sensor distribution or gait segmentation and analysis;

- Research about insole, shoe and eTextile development for feet plantar pressure monitoring; and

- Different goals, such as recognition of environment characteristics.

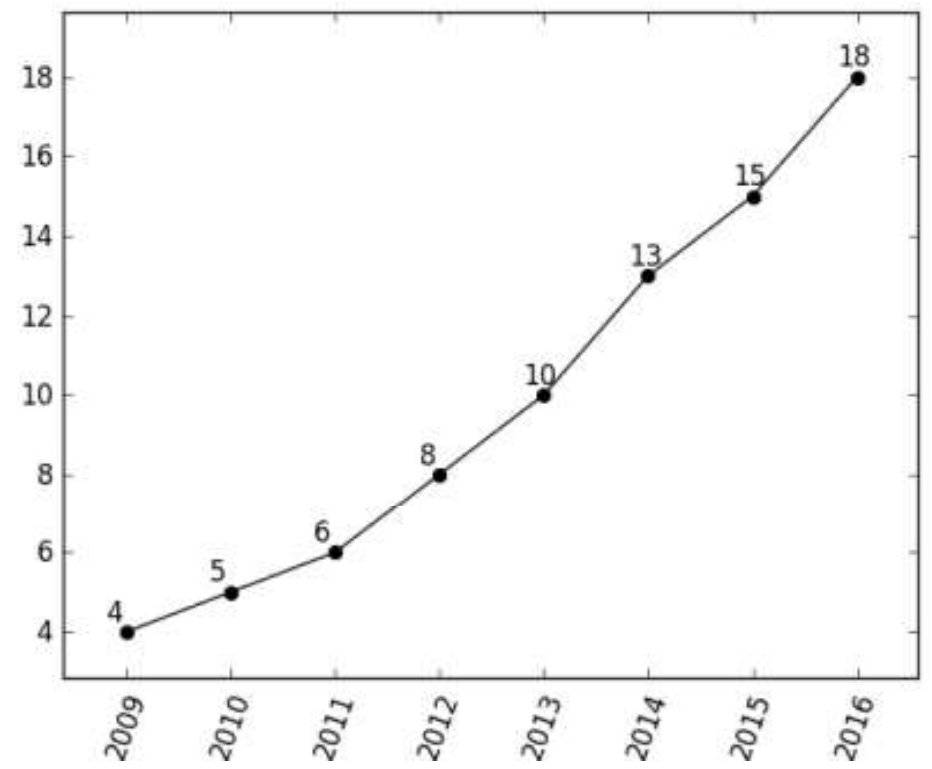

Figure 2.1: Recent surge of interest in investigating the use of embedded sensors in shoes and insoles.

The resulting works were individually analyzed using the proposed exclusion criteria. The remaining 78 works may be grouped into three distinct research categories: (i) user feedback and performance assessment: works focused on the assessment of the quality of execution of an activity to provide feedback to individuals; (ii) activity recognition: works focused on the recognition between activities of an activity model; and (iii) prevention or treatment of injuries and diseases: works that, regardless of focus on recognition or quality assessment and feedback, aimed at preventing, or helping prevent, injuries and diseases. In addition to the works retrieved during the literature review, we contacted experts and asked for suggestions of related research. The work presented in [57], though excluded by the applied criteria, was recommended by an expert. 


\section{1}

\section{User Feedback and Performance Assessment}

The study presented in [57] uses the off-the-shelf Microsoft Kinect device to evaluate posture and analyze users movement, aiming at helping users assess and correct their movements during a CrossFit training session. It is based on four key points: (i) individual monitoring, (ii) audible feedback, (iii) detailed report and (iv) non-invasive monitoring. The researchers developed a system based on the Kinect Sensor and the Skeleton Basics-WPF application to recognize particular movements, monitoring the variation of the joint angles and sending instantaneous audible feedback to users correct their postures. One major limitation of this study is the focus on only one exercise, the thruster, within a restricted context. In addition to that, the methodology employed is not capable of measuring GCFs, a key factor in assessing the risk of RSI, as discussed in chapter 1 . Other limitations of this study are (i) the use of image processing equipment and techniques that are not adequate to monitor groups of practitioners, (ii) the absence of detailed information regarding how the benchmark movement was defined, and (iii) the audible feedback, inadequate for CrossFit ${ }^{\mathrm{TM}}$ classes by reason of practitioners being typically exposed to high sound levels for motivational purposes. The study suggests that the developed application provides a coach-like feedback that can be used to substitute such an expert.

The works proposed by [14] and [3] use upper and lower body accelerometer data to provide, respectively, feedback to swimmers and rowers. In [14], a sensor system is used to extract lap times and stroke counts for each lap of the pool, achieving good overall results. However, in [3], even though the experiment was successfully conducted by providing users with immediate feedback, no improvements were found for performance-related parameters. Another work focused on rowers collects femur and lower back kinematic data through an accelerometer-based body sensor network (BSN) [47]. The proposed system was used, alongside optical tracking, to distinguish between good and poor rowing techniques. The study conducted in [19] devises a wearable system using FSR pressure sensors positioned on the shoe's soles to acquire raw sensor data, similarly to most surveyed works. Instead of using the common standing-walking-running activity model, it maps user's activities into dancing steps: ball taps, heel taps, forward steps, and backward steps. Each dancing step is matched to music beats, and the difference between the performed and the expected step is given to the participants in real-time auditory feedback. Also, in [7], an experiment was conducted with professional swimmers to help improve their performance. The researchers used acceleration sensors to mon- 
itor relevant swimming parameters for a continuous performance evaluation, while also offering visual, audio, and haptic feedback to swimmers. After testing four feedback modes, visual signs were chosen for their fastest reaction time. In the ongoing work presented by [63], an experiment is being conducted with professional skiers aiming at improving the trainer-athlete relationship. Through the usage of wearable sensors and visualization software, participants were able to share their observations and impressions. Another work aiming at benefiting athletes with immediate feedback during training is presented by [6]. The researchers propose feedback systems for rowing, table tennis, and biathlon professional athletes, providing detailed hardware information.

\section{2}

\section{Activity Recognition}

Common movement activities, such as walking, sitting, and standing up, are the most common type of activities studied in this research group. Many works, such as [16], [22], [42], [19] and [18], rely on plantar Forcesensitive Resistors (FSR) pressure sensors to classify user activity according to a previously elaborated activity model. Other works, such as [31] and [62], rely on inertial motion units (IMUs) located on the user's feet for that purpose. Sensor fusion - FSRs and IMUs - is employed by works such as [89], [80], [39] and [56], achieving good overall results. Only a few of the surveyed works used sensors other than ground contact force (GCF) sensors and IMUs, such as infrared sensors [46] or capacitive sensing technology [35] and [61]. The work in [46] presents a novel approach by using an infrared sensor positioned onto the shoe's surface and machine learning models to classify among six activities. It is also the only work presenting a comparison between accuracy results using one or two shoes. In [35] researchers used capacitive sensing technology, placing the sensors on the user's chest, leg, and foot to detect movement and classify among five activities. The reason to use capacitive sensing technologies is its accuracy at low speeds and tiny step lengths, typical of the elderly and patients with pathological conditions that participated in the study, contrary to accelerometer-based detection that can provide high deviations. Capacitive sensing technology is also used in [61], where the researchers use a smart insole to identify users and, above all, distinguish between floor types. Differently, [95] proposes a novel approach using ultrasonic sensors in a wearable platform to monitor lower limb movements and patterns. Although it is an ongoing work, the results show that ultrasonic systems may be successfully used for gait analysis in running and jogging. The researchers in [59] use in-shoe wearable-based sensor technology for gait and turning 
assessment of patients with postural instability due to to Parkinson's Disease. The wearable-based sensor technology used in their experiment allowed for static and dynamic posturography in clinical and home environments - an important assessment for orthopedic diagnosis. In [13], researchers investigate the use of two synchronized insoles for automatic gait phase segmentation using 64 plantar pressure sensors each, showing results in line with those obtained using other methods such as [49]. The study presented in [49] criticizes the use of FSRs, supported by a brief discussion about their low durability and non-linear responses. As a novel alternative to FSRs, the researchers propose the use of four air pressure sensors - air bladders - in each insole.

Some studies positioned extra sensors in other places beyond the user's feet, such as [101] and [94]. In [94], sensors are also placed onto the user's arm, thigh and waist, obtaining almost $100 \%$ activity classification accuracy, although with a restricted activity model comprising walking, sitting down and standing up. In [101], one IMU is placed on the user's waist to help classify activity among zero displacement activities, transitional displacement activities and strong displacement activities. All activity recognition works discussed in this section use very similar activity models comprising sitting, standing, running, walking and slope-walking activities. Henceforth, the main difference being these works is the machine learning algorithms applied and the context of the experiments. Two of the activity models proposed by the works of this section, [100] and [55], are more comprehensive than the prevailing standing, walking and running activity models. Both relied on sensor fusion techniques to classify user's activities and obtained promising results. In [100], the researchers used four plantar FSR pressure sensors and an IMU capable of 9 degrees of freedom motion sensing on the user's smartphone. They were able to classify activity as walking, cycling, bus-passenger, car-passenger and car-driving, using decision tree machine learning techniques. The work in [55] combined a 48-sensor FSR array and an IMU capable of 9 degrees of freedom motion sensing within each insole to predict activities with $99 \%$ accuracy. Another major contribution of those studies is their activity model that includes transitions between ADLs, such as descending stairs to walking or walking to sitting down, in addition to the activities themselves.

The work in [70] presents an extensive research using wearable sensors to understand the best signal processing, sensors and classification methods for classifying health-enhancing physical activities such as walking, running and cycling. It discusses how each activity is best characterized and what sensors should be used to recognize them. Likewise, in the work proposed by [23], activity recognition using wearable sensors provides lifestyle feedback regarding 
health-enhancing physical activities. However, contrary to the previous study, this one focuses on non-lab activities and sports in non-monitored settings. Considering the proposed activity model, results showed that using both unsupervised and supervised data for machine learning yields similar results to using only supervised data. The work in [50] proposes an optimal sensor set for gait identification of patients with dropped foot for clinical evaluation. This sensor set consists of three IMUs capable of six degrees of freedom, placed onto users thigh, shank and foot of the impaired leg. The study identifies the best sensor orientations and attachment positions, whilst accurately identifying gait events. Finally, it suggests that the system could be used to analyze other walking conditions during daily activities.

Many of the surveyed studies, conducted in the recognition of activities, were related to healthcare and well-being. The theme of stroke rehabilitation is being investigated, in recent years, by many researchers, such as in [99], that measures the activity level of people with stroke, and in [21], that recognizes activities and postures to provide behavioral feedback to patients recovering from a stroke. Although the majority of the works focused on healthcare and well-being, some, such as [27], investigated the use of foot-based gestures, named foot plantar-based (FPB) gestures, to control computing devices. Shoebased wireless sensor platforms developed specifically for medical studies, such as the SmartStep [41], were employed by many other health-related studies. In [40], the platform was used to develop an Android application to capture data from the wearable device and provide real time recognition of a small set of activities. In [79] and [81], the SmartShoe platform is used for energy expenditure estimation after the classification of the activities performed by the user, and in [82] it is further used to predict body weight. The same platform is then used by [29] and [28] to identify activity levels and steps in people with stroke.

\section{3}

Prevention and Treatment of Injuries and Diseases

The prevention and treatment of injuries and diseases is the second most prevalent theme of research found in the HAR literature review, after gait analysis. The research presented in [53] and [54] recognizes that the first step to reduce caregivers' risk of overexertion injuries is to identify patient handling activities (PHA). It extends the work presented in [55], which uses an eTextile fabric with 48 plantar pressure sensors and an IMU capable of nine degrees of freedom, to detect user activity and identify awkward postures that might lead to injuries. Despite the complexities of the interaction between users and 
loads (e.g., patients and instruments), both studies show promising results.

In works such as [2] and [5], researchers propose an accelerometer-based wearable framework for recognizing athletes' activities in outdoor training environments. They aim at identifying (i) potential injury or performance determining factors, (ii) users in the early stages of a developing injury, and (iii) a predisposition to injury based on movement patterns. In this work, the researchers performed an experiment to monitor thigh and shank movement and posture during jogging, achieving good overall results. In [67], the researchers present a pair of shoes that offer low-cost balance monitoring non-lab environments. The shoes use features identified by geriatric motion study experts to monitor balance and predict fall risk, demonstrating the feasibility of a model of instability assessment. This lightweight smart shoes platform is based on the MicroLEAP wireless sensor platform [4], and uses an IMU and FSR pressure sensors embedded inside each insole for data acquisition.

\section{4}

\section{Literature Review Discussion}

To better evaluate patterns, common strengths and weaknesses of the surveyed works, we collected, whenever available, the following research data:

- Number of activities in the activity model;

- Number of subjects;

- Number of data samples collected; and

- Test technique and results.

The collected research data, addressing research questions 2, 3, 5 and 5, is summarized on Table 2.1. In the interest of comparison, the metadata of this work was added to the first row of the table. Some works on this table achieved multiple different accuracy results, since they addressed multiple challenges. This table presents only the accuracy results of activity recognition classifiers. All dataset size is rounded to the nearest thousandth.

The measuring of GCFs is the most prevalent approach used by the surveyed works for the task of recognizing user activity, being present in 16 of the 24 studies, while the second most common approach, present in 12 of the 24 studies, is the use of IMUs. Considering the IoT devices presented in the literature, two characteristics impair their reproducibility: (i) the lack of information about the IMUs orientation and (ii) the absence of sensor model information or specification. Most of the works analyzed, 22 out of 24, provided detailed information regarding the models of their activity classifiers, but only 


\begin{tabular}{|c|c|c|c|c|c|}
\hline \multicolumn{6}{|c|}{ Metadata Summary } \\
\hline Author & Model & Subjects & Data & Technique & Results \\
\hline de Pinho et al. & 13 & 12 & $1 \mathrm{M}$ & 10-Fold & $\approx 87 \%$ \\
\hline Crea $[13]$ & 5 & 5 & - & Leave-one-out & $\approx 96 \%$ \\
\hline De Santis [16] & 4 & - & - & - & - \\
\hline Doppler [18] & 3 & 4 & $36 \mathrm{k}$ & 10-Fold & $99.6 \%$ \\
\hline Drobny $[19]$ & - & 16 & - & - & - \\
\hline el Achkar [22] & 3 & 5 & - & - & $93.4 \%$ \\
\hline Fukahori [27] & 15 & 20 & - & - & $\approx 99 \%$ \\
\hline Ghobadi [31] & 3 & 3 & - & - & $99.7 \%$ \\
\hline Haescher [35] & 5 & 13 & $9 \mathrm{k}$ & Leave-one-out & $\approx 89 \%$ \\
\hline Holleczek [42] & 2 & 1 & - & 4-Fold & $100.0 \%$ \\
\hline Jiang $[46]$ & 6 & 4 & - & 10-Fold & $\approx 94.0 \%$ \\
\hline Kong [49] & 6 & 1 & - & - & - \\
\hline $\operatorname{Lin}[55]$ & 8 & 8 & - & - & $86.6 \%$ \\
\hline $\operatorname{Lin}[56]$ & 5 & 10 & $2 \mathrm{k}$ & - & $99.0 \%$ \\
\hline Matthies [61] & - & 13 & - & 10-Fold & $90 \%$ \\
\hline Tang [89] & 6 & 9 & $21 \mathrm{k}$ & Leave-one-out & $99.0 \%$ \\
\hline McCarthy [62] & 4 & 13 & - & - & $90.0 \%$ \\
\hline Edgar $[21]$ & 3 & 3 & - & - & $99.0 \%$ \\
\hline Noshadi [67] & - & 12 & - & - & - \\
\hline Sazonov [80] & 6 & 9 & - & 4-Fold & $98.0 \%$ \\
\hline Sazonov [79] & 15 & 15 & - & Leave-one-out & $95.0 \%$ \\
\hline Ugulino [94] & 4 & 4 & $165 \mathrm{k}$ & 10-Fold & $\approx 99.0 \%$ \\
\hline Zhang [99] & 8 & 12 & - & Leave-one-out & $\approx 80 \%$ \\
\hline Zhang [100] & 5 & 10 & $7 \mathrm{k}$ & 10-Fold & $90.0 \%$ \\
\hline Zhu [101] & 3 & 1 & - & - & - \\
\hline
\end{tabular}

Table 2.1: Literature review metadata: methods and results.

12 studies detailed the test techniques used in the activity classifiers - 7 of which used k-fold cross validation and 5 used leave-one-out. Although most studies state that tests were performed, they provide no detailed information about these tests. The success rate of ADL recognition of the surveyed works fell into the $80 \%$ - $100 \%$ range. All works analyzed in this section use the model of gait phases proposed by [72]. Addressing research question 1, the surveyed works' approach to activity execution is binary, considering a performed activity as either correct or incorrect. No grading or qualitative analysis of execution mistakes were discussed in the surveyed works.

It was also observed that 21 out of 24 works provided information on the number of participants, but only 6 of the 24 studies provided information on the dataset size. The main issue detected in the literature review is the absence of publicly available datasets, impairing that way the understanding of the results. Knowledge of datasets is especially important to assess works that use similar (i) sensor selection, (ii) sensor placement, and (iii) activity 
models. As discussed in [101] and [98], dataset disclosure is crucial for benchmarking purposes, given that classification algorithms rely heavily on datasets. Beyond dataset size, sample rates and time window durations also affect the understanding of the results. Only 16 of the 24 surveyed works provided information on the sample rates, and 8 of those 16 also provided information on the time window size. The prevailing suggestions for future works and contribution found in the literature are to (i) increase the data set through longer data collection intervals and the diversification of participant's profiles and (ii) adapt the activity model to a specific challenge, such as helping patients to avoid falls. As noted in [73], the prevailing suggestions for future works and contribution found in the literature are (i) the increase the data set through longer data collection intervals and the diversification of participant's profiles, (ii) the improvement of the selected classification algorithms, and (iii) the adaptation of the activity model to a specific challenge, such as helping patients to avoid falls, instead of a more broad task, such as monitoring patients' balance. To make the literature review presented in this section replicable, we provided (i) the search query string, (ii) the exclusion criteria, (iii) information about the selection process and (iv) the surveyed publications in RIS format in the following web address: https://goo.gl/6ozm26.

The challenge of adequately positioning the GCF sensors is recognized as a very important factor by studies such as [83] and [58], although it is not thoroughly addressed by any of the surveyed works. To understand the literature recommendations of sensor deployment before the development of the IoT wearable device, we collected, whenever available, the types, quantities and locations of the sensors employed by the surveyed works. No work addressed the sensor mix choice besides stating that its selection is commonly found throughout the literature. The sensor data, addressing research question 6 , is summarized on Table 2.2. In the interest of comparison, the metadata of this work was added to the first row of the table. The table is divided into three segments: (i) the upper segment summarizes works based on GCF sensors, predominantly FSRs, (ii) the middle segment summarizes works based on inertial sensors, and (iii) the bottom part summarizes works that employed sensor fusion. No work provided detailed information regarding sensor positioning. 
Summary of sensor selection and positioning

\begin{tabular}{lll} 
Author & Sensors & Positioning \\
\hline de Pinho et al. & FSR, IMU \& IR & Insole, Shoe \& Knee \\
\hline Crea [13] & FSR & Insole \\
De Santis [16] & FSR & Insole \\
Drobny [19] & FSR & Insole \\
el Achkar [22] & FSR & Insole \\
Fukahori [27] & FSR & Socks \\
Holleczek [42] & FSR & Socks \\
Lin [55] & FSR & Insole \\
Zhu [101] & FSR & Shoe \& Waist \\
Kong [49] & GCF & Insole \\
\hline Ugulino [94] & Accelerometers & Waist, Thigh, Arm \& \\
& & Ankle \\
McCarthy [62] & Gyroscope & Shoe \\
Doppler [18] & IMU & Insole \& Shoe \\
Ghobadi [31] & IMU & Shoe \\
\hline Edgar [21] & FSR, IMU & Insole \\
Lin [53] & FSR, IMU & Insole \& Shoe \\
Tang [89] & FSR, IMU & Insole \\
Zhang [99] & FSR, IMU & Insole \& Shoe \\
Zhang [100] & FSR, IMU & Insole \& External \\
Noshadi [67] & FSR, Gyroscope \& & Insole, Shoe \& External \\
& Accelerometers & \\
Sazonov [79, 80, 81, 82] & FSR \& Accelerometers & Insole and Shoe \\
Jiang [46] & IR & Shoe \\
Matthies [61] & Capacitive Sensing & Insole \\
Haescher [34, 35] & Capacitive Sensing & Chest, Leg and Insole \\
& &
\end{tabular}

Table 2.2: Literature review metadata: sensor positioning. 


\section{3 IoT Device Prototype}

In this chapter, we present the wearable IoT device prototype used to collect functional training data. To allow for the reproduction of this research, we provide detailed hardware information - types, quantities and models for each component.

Since battery lifetime is a major concern for studies outside of a laboratory environment and the participants came from diverse technological backgrounds, the key design goals were to develop a low power consumption device capable of extended operation that was easy to use during the experiment. Aiming at a user base as diverse as possible, we followed the prototyping principles discussed in [73] and [74]. To reach these goals, we developed a wearable Io $\mathrm{T}$ device that (i) uses only the power button, (ii) requires only one functional foot to collect user data, and (iii) transmits data for external processing. We opted to use a single insole in the experiment to reduce prototyping costs and to broaden our user base, since the literature suggested that the loss of information when compared to experiments collecting data from both feet is minimal [46] and [75]. The wearable device comprises three components: (i) an US men's size 8.5 (see Table 3.1) insole that houses the plantar pressure sensors, (ii) an external protective case that houses the microcontroller and the foot sensor array, and (iii) an external protective case that houses the knee sensor array. The use of an US men's size 8.5 insole allows for - although with minor discomfort felt at extreme ranges - the participation of volunteers within the US men's size 7.5 to the US men's size 10.5 range.

The insole employs six GCF sensors following the literature's recommendations for placing discussed in [83], [72] and [60], in addition to the lessons learned from the prototypes presented in [73], [76] and [74]. We used the FSR 402, by Interlink Electronics [45]- a PTF (Polymer Thick Film) device that exhibits a decrease in resistance as the force applied to its active surface increases - and Amphenol Clincher Connectors, by FCI [24], to avoid melting or distorting the silver traces of each sensor. Given that each FSR needs a static resistor to create a variable voltage for the microcontroller's Analog to Digital Converter (ADC) inputs, we placed six $10 K \Omega \frac{1}{4} W$ resistors inside the insole one resistor next to each FSR. GCF sensor positioning is shown in Figure 3.1. 
Shoe Size Comparison Chart

\begin{tabular}{llllllll} 
USA and Canada, Men & 7 & 7.5 & 8 & 8.5 & 9 & 10.5 & 11.5 \\
\hline Europe & 39 & 40 & 41 & 42 & 43 & 44 & 45 \\
United Kingdom, Men & 6.5 & 7 & 7.5 & 8 & 8.5 & 10 & 11 \\
United Kingdom, Women & 6 & 6.5 & 7 & 7.5 & 8 & 9.5 & 10.5 \\
USA and Canada, Women & 8.5 & 9 & 9.5 & 10 & 10.5 & 12 & 13 \\
Japan, Men & 25 & 25.5 & 26 & 26.5 & 27.5 & 28.5 & 29.5 \\
Japan, Women & 24.5 & 25 & 25.5 & 26 & 27 & 28 & 29 \\
Mexico & 5.5 & 6 & 6.5 & 7 & 7.5 & 9 & 10 \\
Brazil & 37 & 38 & 39 & 40 & 41 & 42 & 43 \\
Australia, Men & 6.5 & 7 & 7.5 & 8 & 8.5 & 10 & 11 \\
Australia, Women & 8.5 & 9 & 9.5 & 10 & 10.5 & 12 & 13
\end{tabular}

Table 3.1: Comparison between US men's sizes and other standards.

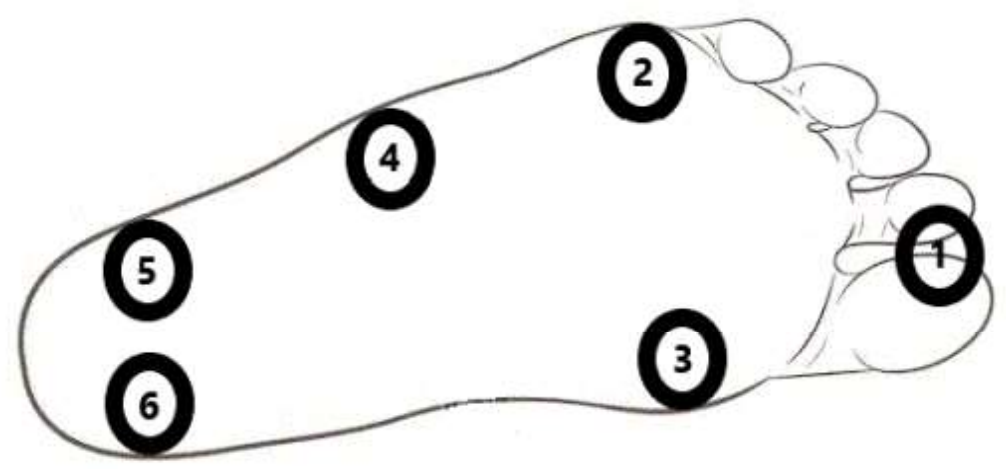

Figure 3.1: GCF sensor positioning.

The main component of the foot external protective case is the Electron, a 3G-enabled microcontroller from Particle.io [71], shown in Figure 3.2. This microcontroller was developed for cellular-connected electronics projects that require low-bandwidth, and contains all inputs, outputs and communication protocols necessary for the presented IoT wearable device prototype. The component collects data from the foot sensor array and streams the data to the remote database. For the accelerometer, gyroscope and magnetometer sensors responsible for monitoring the feet posture and movement, we used the SparkFun 9DoF IMU LSM9DS1 Breakout [88], a system-in-package component that houses a 3-axis accelerometer, 3-axis gyroscope, and 3-axis magnetometer sensor array that is capable of digital communication with the Electron microcontroller. The LSM9DS1, shown in Figure 3.3, is used to measure the key movement properties of the foot - angular velocity, acceleration, and heading - in three dimensions. The barometer selected for the experiment is the MPL3115A2 [68], by Freescale Semiconductor, a low power, high-precision altitude and pressure, shown in Figure 3.4. For the distance sensor we used 
two of the RFD77402 3D ToF (Time of Flight) by Simblee [84], a low-cost accurate sensor that allows millimeter readings up to two meters, shown in Figure 3.5. The RFD77402 uses an infrared VCSEL (Vertical Cavity Surface Emitting Laser) module to measure the amount of time the emitted light takes to bounce off a target.

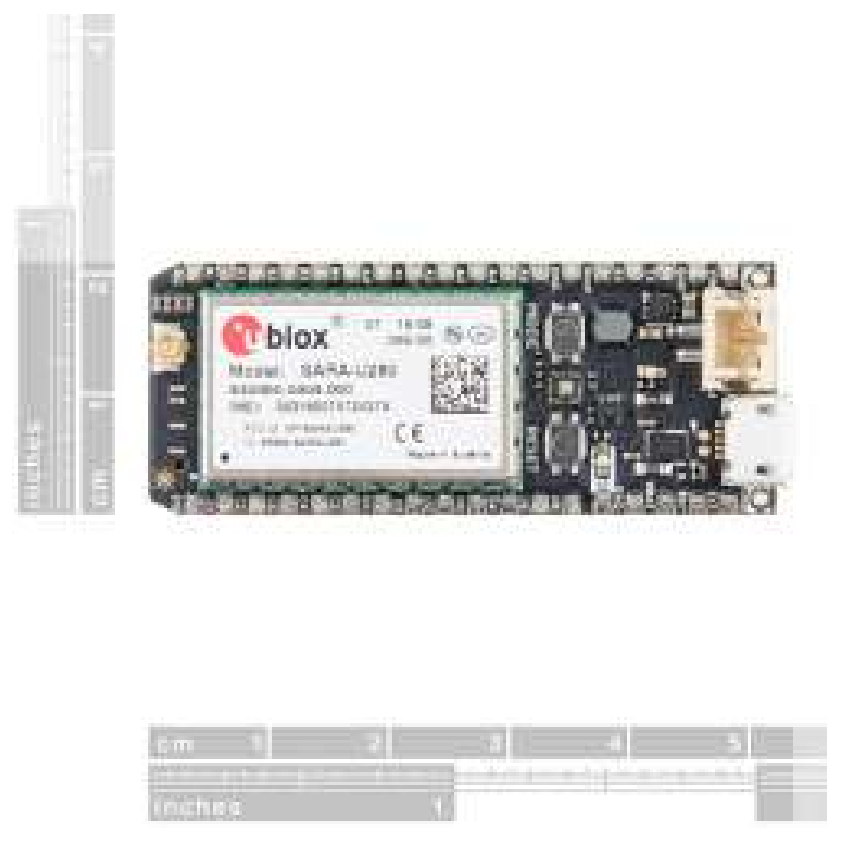

Figure 3.2: The Particle.io Electron.

The Electron microcontroller and its board, along with the MPL3115A2 and RFD77402 sensors mentioned above, were positioned in the ABS 3D printed external protective case, shown in Figure 3.6. The prototype is powered by a 2,200 milliampere hour (mAh) lithium ion battery pack by Sparkfun Electronics [87], allowing for an easier, faster replacement and improved usability.

The knee external protective case houses a second SparkFun 9DoF IMU LSM9DS1 Breakout to measure the movement properties of the knee, and is connected to the foot external protective case by a flexible cable, shown in Figure 3.7.

The software model used in this work is based on the model proposed in [73] and [75], comprising two components: the embedded software running on the microcontroller and the application server. The microcontroller software is responsible for (i) acquiring the raw sensor data, (ii) structuring the data in JavaScript Object Notation (JSON) format, and (iii) transmitting the structured data over $3 \mathrm{G}$ to the application server. The application server is responsible for processing and logging the streamed data to Firebase 


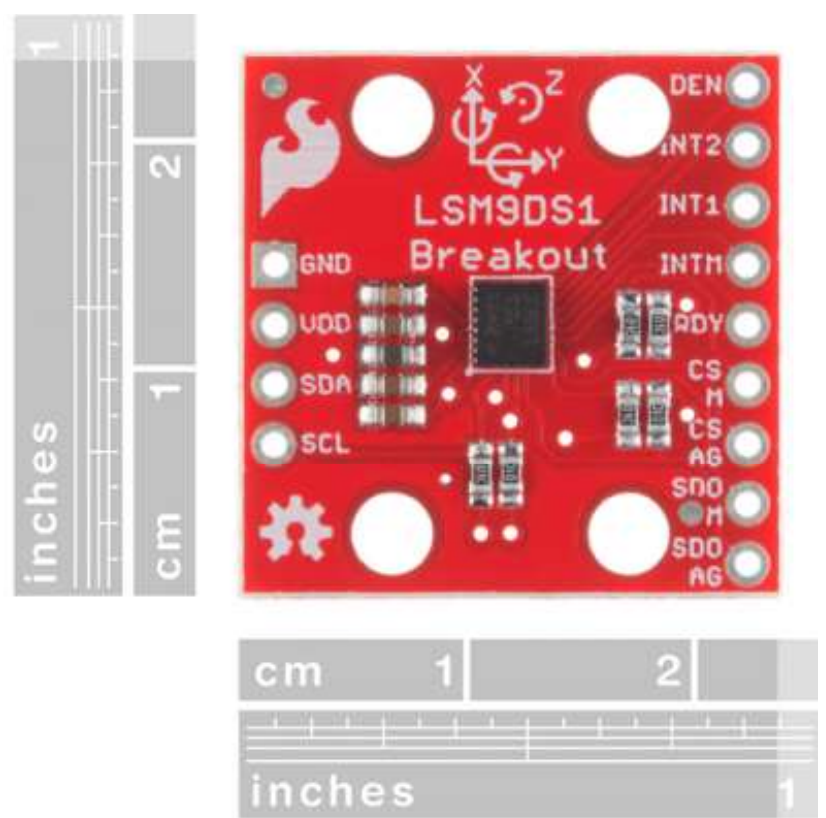

Figure 3.3: The LSM9DS1 IMU.

Realtime database - tasks not suitable for the embedded microcontroller due to its hardware limitations. The Firebase Realtime Database is a cloudhosted database supported by Google that stores data in the JSON format and synchronizes to every connected client. The authors made available the complete and commented source code of the application server in [73].

\section{1}

\section{Analysis of the Sensor Mix}

This section analyses the sensor mix selection of the IoT device prototype used in this work. As discussed in chapter 2, no work addresses sensor selection choices and concerns. In [74], we conducted an experiment to understand the relevance of the most pervasive sensor types in the literature for the building of HAR classifiers for the most commonly-found activities - walking straight $(2 \mathrm{~km} / \mathrm{h})$, walking slope up $(2 \mathrm{~km} / \mathrm{h})$, walking slope down $(2 \mathrm{~km} / \mathrm{h})$, slow jogging $(6 \mathrm{~km} / \mathrm{h})$, slow jogging slope up $(6 \mathrm{~km} / \mathrm{h})$, slow jogging slope down $(6 \mathrm{~km} / \mathrm{h})$, ascending stairs, descending stairs and sitting. The experiment employed the IoT device prototype previously described, built with a sensor array containing all sensors found in the surveyed works. We collected data from twelve participants to build and validate a baseline model - a model that makes use of all sensor raw and derived features. Different learning strategies were experimented to build the classifier for our 9-activities activity model, resulting in the selection of the Random Forest Algorithm, given that its 

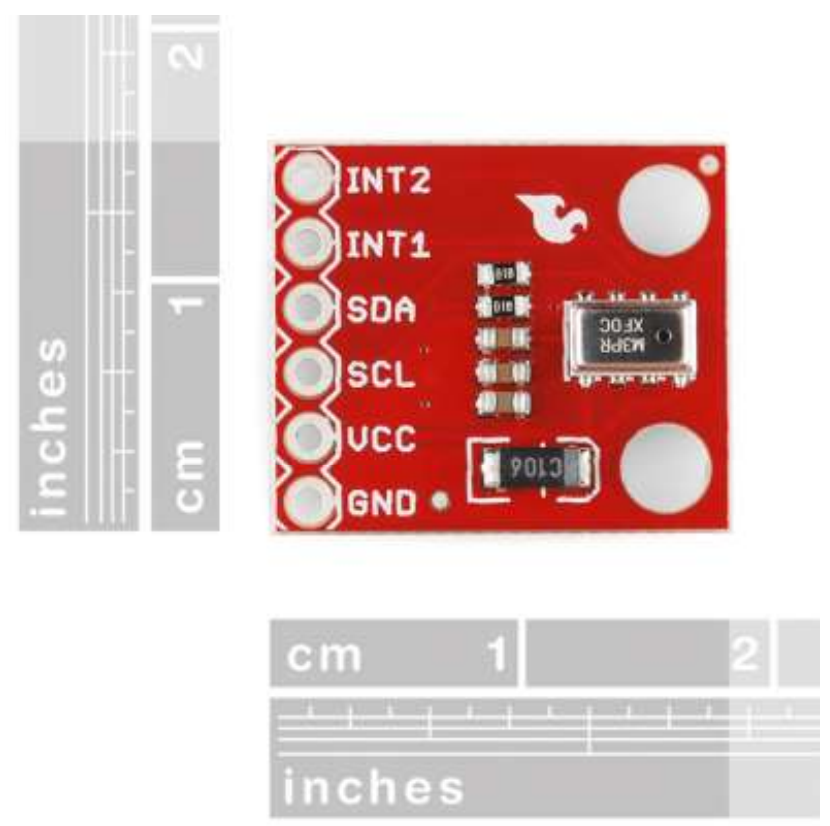

Figure 3.4: The MPL3115A2 barometer.

average performance of $91.26 \%$ was superior to the other ones. For validation, we used the Leave-one-out Cross Validation, since this method guarantees that both training and test splits do not share any example data. In total, 12 features were utilized to build the classifier: 2 axis of the gyroscope, 2 axis of the magnetometer, 1 axis of the accelerometer, 4 FSRs, 2 Euler angles and the cumulative difference between samples of the barometer. The features selected for the baseline classifier provided valuable insights for the next step, the analysis of sensor type relevance. After experimenting several time window sizes during the classifier construction, we decided to use a $0.3 \mathrm{~s}$ one based on our model validation results, registering an accuracy improvement of about $19 \%$ when contrasting the selected window $(0.3 \mathrm{~s})$ and the largest time window experimented (2.0s). Despite not taking any measures to address FSRs' drift over time, as discussed in [74], the overall classifier accuracy was satisfactory - achieving top quartile performance when compared to the surveyed works.

Following the building of the baseline HAR classifier, we were able to investigate the relevance and correlation of a feature with the average classifier accuracy, for each sensor type. We used Hall's algorithm [37] based on correlation with its default "Best Fit" configuration, a backtracking greedy strategy. This method was selected based on its superior performance when compared to the other feature selection methods found in the surveyed works. The work in [94] also uses this same method to reduce feature redundancy and still achieve better-than-average results. Based on the results commonly 

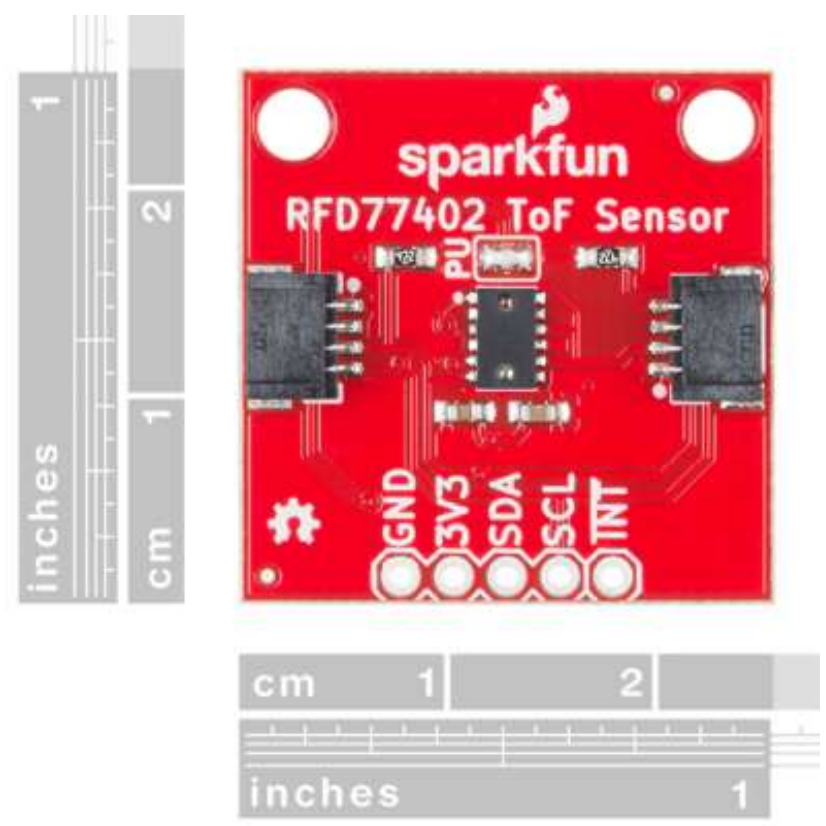

Figure 3.5: The RFD77402 distance sensor.

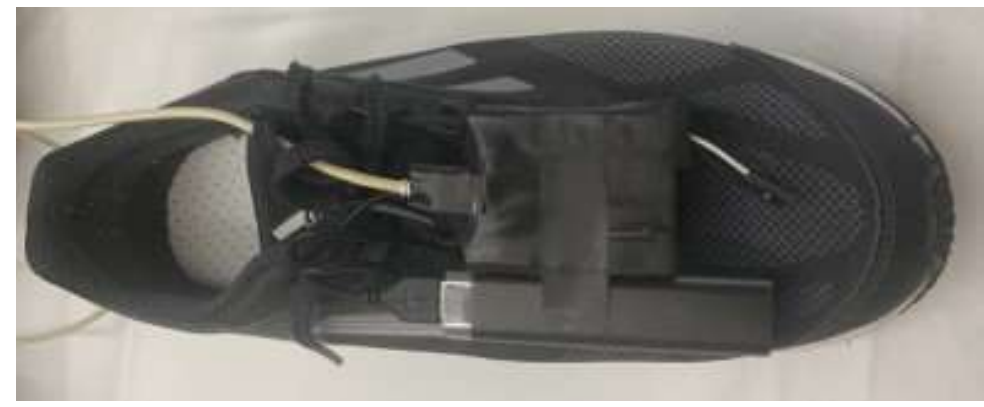

Figure 3.6: Shoe external protective case.

found in the literature, we already expected to see gyroscope, accelerometer and FSR features showing high correlation and being used in the building of the classifier. However, the results achieved by Hall's algorithm suggested which features were relevant and to what extent. Considering the baseline model, the distance sensor showed a very low correlation and was not used in the building of the classifier. Although distance sensors were successfully employed by works such as [46], when presented with a broader sensor selection the classifier did not make use of it. FSRs 4 and 6 showed a medium to low correlation and were not employed, while the remaining FSRs showed a high correlation. Considering that some of the surveyed works also used between three to four FSRs with similar positioning, further investigation could lead to evidence as to where to position the sensors for better recognition accuracy 


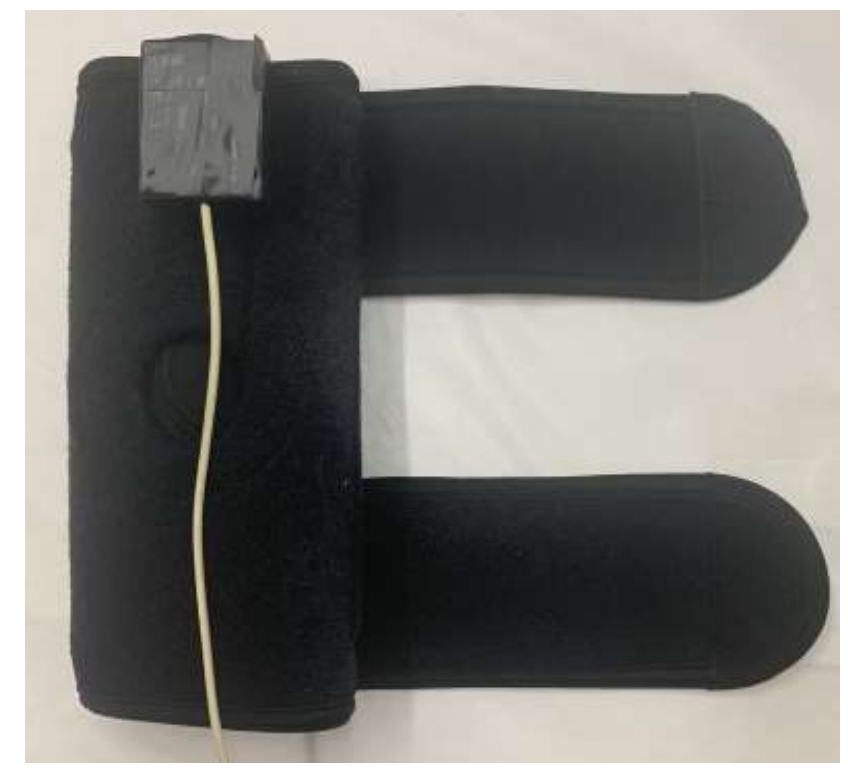

Figure 3.7: Knee external protective case.

\begin{tabular}{ll} 
Sensor & Overall accuracy \\
\hline IMU & $66 \%$ \\
FSRs & $60 \%$ \\
Barometer & $29 \%$ \\
Distance & $17 \%$
\end{tabular}

Table 3.2: Single sensor classifier accuracy.

of the selected activities. Euler angles showed a high correlation and were utilized by some of the surveyed works, making a strong case that they should be evaluated during the feature engineering phase for similar activity models. Finally, the barometer provided the feature with the second highest correlation score, suggesting that this type of sensor should also be evaluated for similar activity models. The topic of feature engineering and its methods are beyond the scope of this research, so we used the features employed by the surveyed works in our analysis.

To assess each sensor, raw and derived features, we built a HAR classifier employing only its share of the total data and the same methods used to build the baseline classifier: (i) Leave-one-out Cross Validation and (ii) Random Forest Algorithm. The average accuracy results are shown on Table 3.2, where all values are rounded to the nearest whole number and ordered by average accuracy.

Following that analysis, we combined the two most successful sensor types, FSRs and IMUs, with all available sensor types. We built and validated a classifier for each combination, and the average accuracy results for the better 


\begin{tabular}{ll} 
Sensor & Overall accuracy \\
\hline IMU (9-axis) and FSRs & $81 \%$ \\
FSRs and Barometer & $71 \%$ \\
IMU (9-axis) and Barometer & $70 \%$ \\
FSRs and Gyroscope & $65 \%$ \\
FSRs and Accelerometer & $60 \%$ \\
FSRs and Magnetometer & $59 \%$ \\
IMU (9-axis) and Distance & $57 \%$ \\
FSRs and Distance & $55 \%$ \\
Distance & $17 \%$
\end{tabular}

Table 3.3: Sensor Fusion classifier accuracy.

performing combinations are shown on Table 3.3, where all values are rounded to the nearest whole number and ordered by average accuracy.

Although the results shown in Table 3.3 take into account the combined FSRs, FSR1 to FSR4, we also evaluated FSR5 and FSR6 for the same combinations, achieving less than $1 \%$ increase in average accuracy. The experimental results show that (i) 9-Axis IMUs, (ii) 4-FSR arrays, and (iii) barometers were the most relevant sensor types for the recognition of ADLs based on feet movement and posture information, when considering a typical activity model with the stand, walk, run and climb stairs activities. Above that, we learned that accuracy can be greatly improved (15\%) by the IMU and FSR sensor fusion, so that approach should be considered in future research if it is needed to (i) differentiate between similar activities or (ii) identify subtle variations of the same activity. Lastly, the barometer sensor allowed for an increase of the average accuracy by more than $10 \%$, making a strong case for its adoption whenever possible. We decided to keep FSR5 and FSR6 for this study, since (i) our goal relies heavily on plantar pressure distribution analysis, (ii) it is not based the typical activity model used in [74], and (iii) we were aiming at recognizing the qualitative aspects of an activity - a challenge still scantly addressed in the literature. 


\section{4}

\section{First Experiment and HAR Classifier Building}

In this chapter we describe the stages followed to develop the HAR activity classifier for movement pattern recognition: planning the experiment, conducting the experiment, acquiring data, processing the acquired data, extracting and selecting features, building and validating the model.

\section{1}

Planning the Experiment

As discussed in chapter 1, the most commonly injured body areas in functional exercise training, in order of occurrence, are the shoulder, the lower back and the knee. Since the research conducted in [73] and [76] allowed for the investigation of the movement patterns and positioning of the lower limbs, it was necessary to understand if data collected from the foot and knee of an individual was adequate to build a movement pattern HAR classifier for functional exercises. During the planning of the experiment, we conducted (i) non-structured open interviews with certified health professionals and CrossFit ${ }^{\mathrm{TM}}$ Level 1 coaches and (ii) surveyed medical research focused on functional training exercises to discern what musculoskeletal aspects are responsible for the most prevalent injuries.

The most important activities in functional training exercise routines are jumping, pulling and pushing [30]. The hip extensors, key to these activities, are the muscles responsible for the straightening out of the hip joint from its flexed position - the hip extension movement [15]. The hip extensors are part of the posterior chain muscle group, a muscle group that comprises the hamstrings, the glutes, and the adductors, shown in Figure 4.1. The musculoskeletal aspects of the posterior chain are related with the most prevalent injuries in functional training, and require the greatest amount of outside input from coaches and training partners - in the absence of outside reinforcement, correct execution form is the first aspect deteriorate [78].

Based on the medical research and the conducted interviews, we selected the following functional exercises to add in our activity model: basic squat, sumo squat, squat hold, basic step up, front lunge, and side lunge. Since the classifier we proposed in [73] performed well, achieving $93.34 \%$ overall accuracy, 


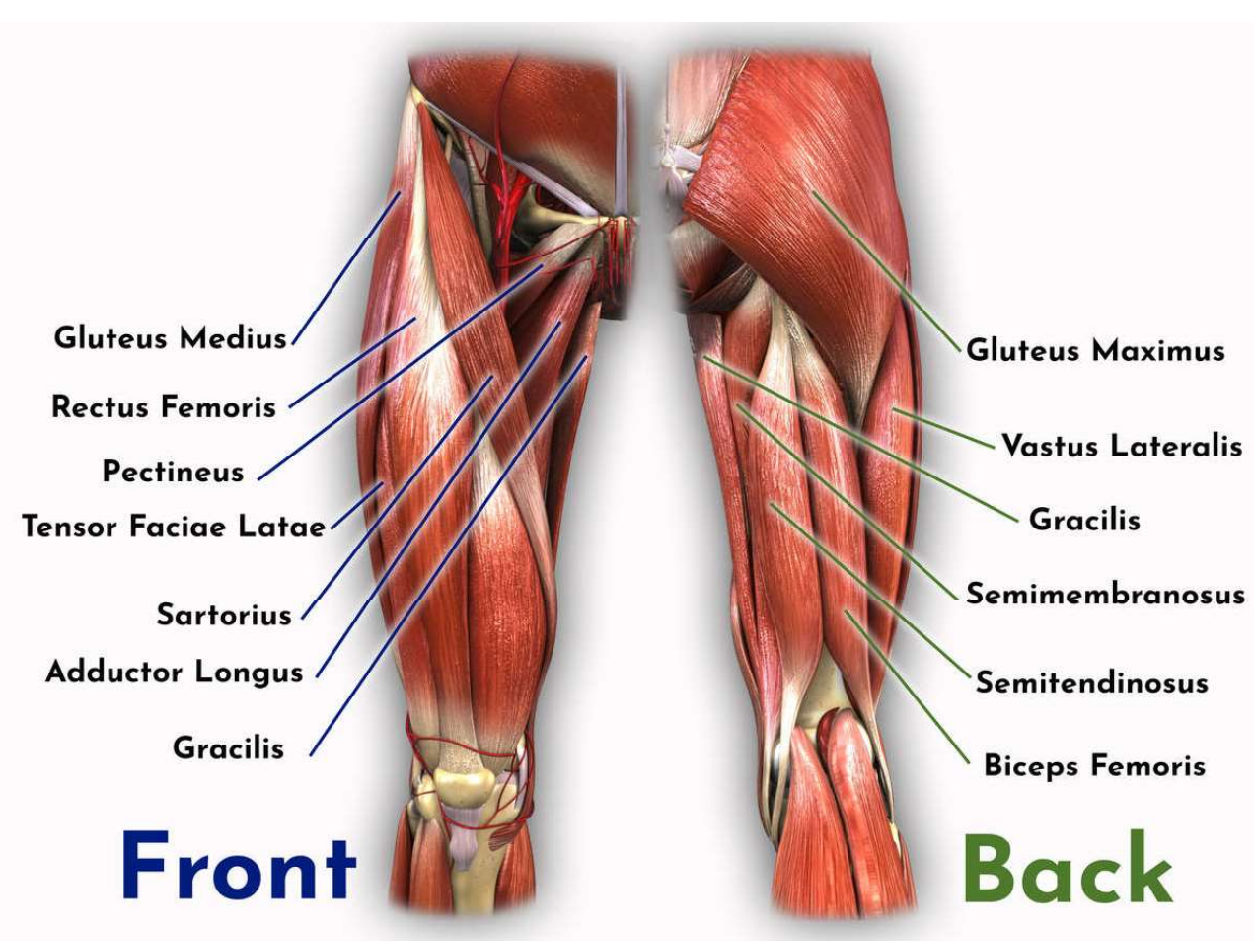

Figure 4.1: Hip Extensors: main and auxiliary muscles [90].

we were confident to extend the original activity model of 6 simple activities to the current activity model of 13 complex activities for this experiment.

The squat, with its variations, is the only exercise in the repertoire of weighted human movement that allows for the direct training of the complex hip drive movement pattern - the active recruitment of the muscles of the posterior chain. [78]. The basic squat is shown in Figure 4.2. In the squat starting position, the individual stands straight with heels placed by shoulder width. The hips and knees face forward, while the toes point outward at an angle of around 30 degrees. Then, the individual slowly bend his knees and pushes his hips backward, as if sitting back into a chair, until his thighs are below parallel with the ground. In the bottom position the back should be inclined at an angle of around a 45 degrees. In the final movement of this pattern, the individual brings his hips up returning to the starting position.

The squat hold follows the same movement pattern, however, the individual keeps the bottom position for an extended amount of time, according the the training regimen. The sumo squat is shown in Figure 4.3. This exercise is a variant form of the basic squat, with two main differences: (i) the toes point outward at an angle of around 45 degrees and (ii) the back, in the bottom position, is kept straight.

The front lunge movement pattern is shown in Figure 4.4. The individual 

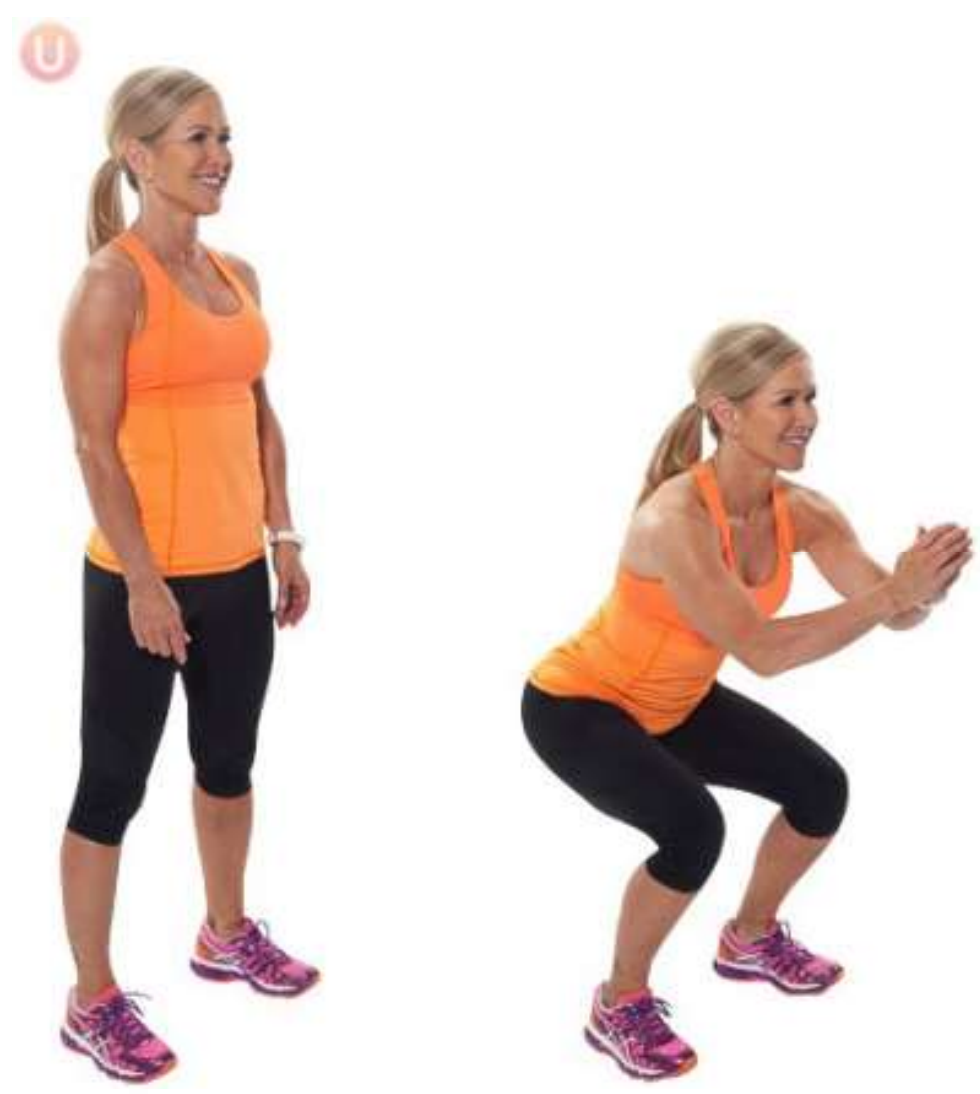

Figure 4.2: Basic squat: start and end positions [26].

stands straight with heels placed by shoulder width and hands either by their sides or placed on their hips. Keeping the torso upright, they inhale as they take a large step forward with one leg and lower the knee of his back leg until it almost touches the floor. Then, the individual exhales as they drive back up with their front leg and step back into a standing position. To complete the full movement pattern, they repeat the lunge with his opposite leg.

The side lunge is a variant of the front lunge, and the movement pattern is shown in Figure 4.5. The individual stands straight with heels placed by shoulder width and hands either by their sides or placed on their hips. Keeping the torso upright, they (i) inhale as they take a large step to one side, (ii) lower their body by flexing their hip and knee and (iii) keep the back leg straight. Then, they exhale as they push themselves back up to the starting position. To complete the full movement pattern, they repeat the lunge with their opposite leg.

The step-up movement pattern is shown in Figure 4.6. The individual stands straight with heels placed by shoulder width and hands either by his sides or placed on his hips. Keeping the torso upright, they inhale as they 

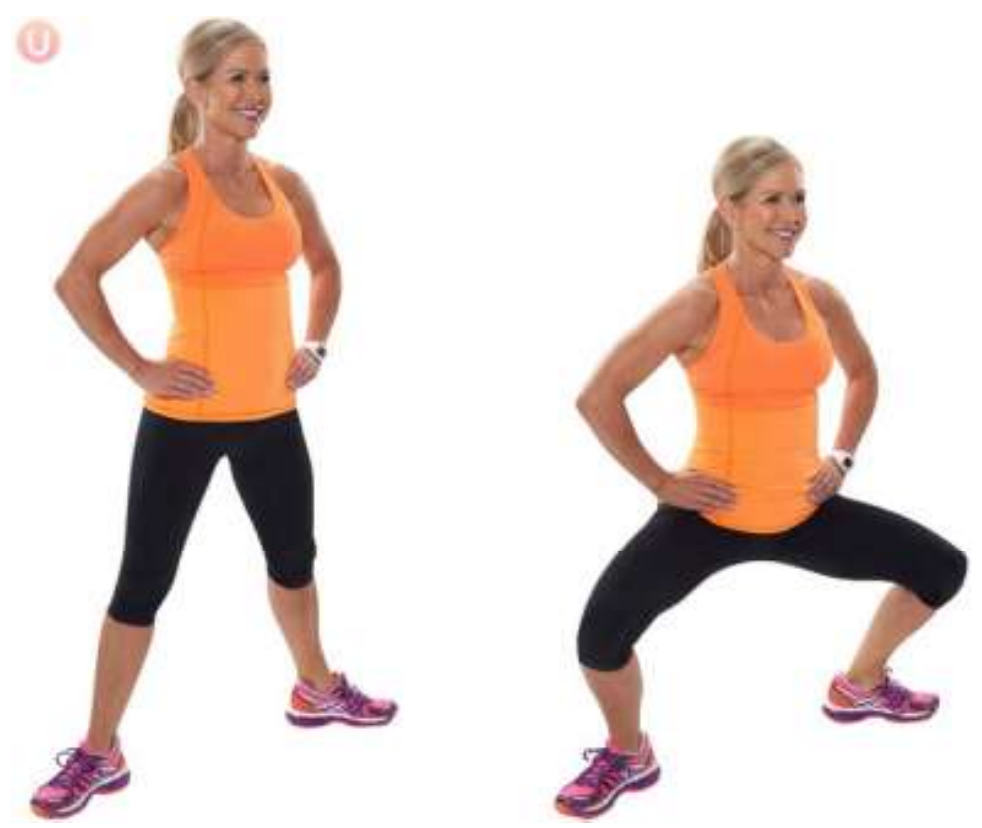

Figure 4.3: Sumo squat: start and end positions [26].

slowly step up onto the bench, leading with their right leg. Then, they bring their feet together on the bench. Next, they inhale as they slowly step down off the bench, leading with their right leg. Lastly, they bring their feet together on the floor. To complete the full movement pattern, they repeat the step-up with their left leg.

These exercises, when performed correctly, are the safest leg exercises for the knees [78]. In addition to that, these exercises aid in the development of the muscle groups that stabilize the knees, mitigating the risk of common injuries and chronic conditions such as knee or back pain. The correct form, when performing these functional training movement patterns driven by the posterior chain, is based on (i) depth of movement, (ii) back positioning, (iii) eye gaze, (iv) knee positioning, and (v) foot positioning.

The depth of the movement characterizes a full extension, with thighs slightly below parallel with the ground. When the individual does not execute the complete extension of the movement, they perform a partial extension. Partial extension stresses the knees and the quadriceps without stressing the glutes, adductors, or hamstrings. The angle restricts the posterior force produced by the hamstrings, needed to oppose and balance the anterior force exerted by the quadriceps and their attachment to the front of the tibia. The difference between a full and a partial extension is shown in Figure 4.7. The result is an anterior shear on the knees and, in addition, the probability of a patellar RSI [78]. According to the information collected during the 

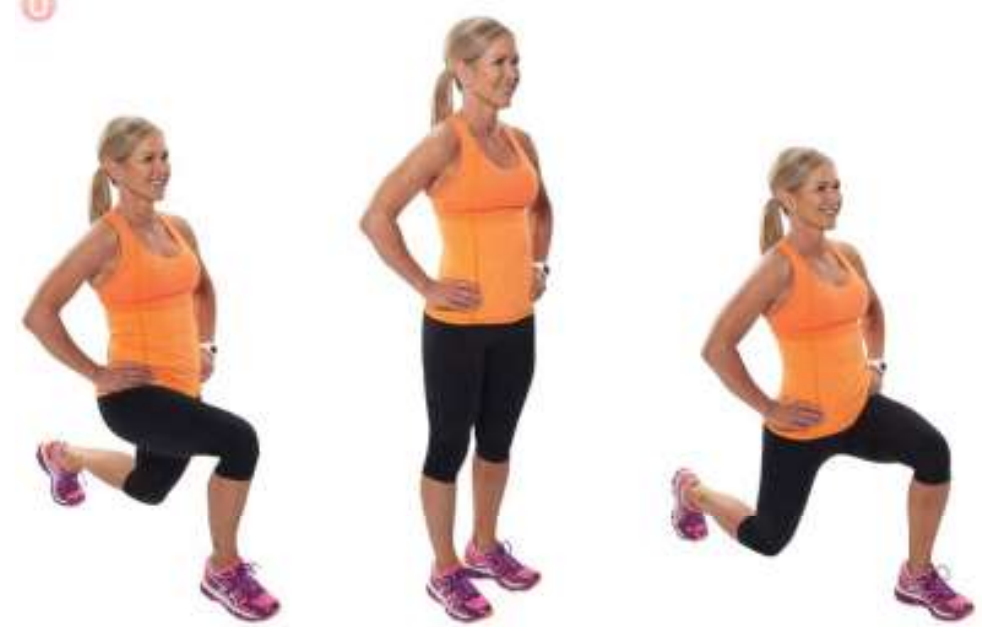

Figure 4.4: Front lunge: start and end positions [26].
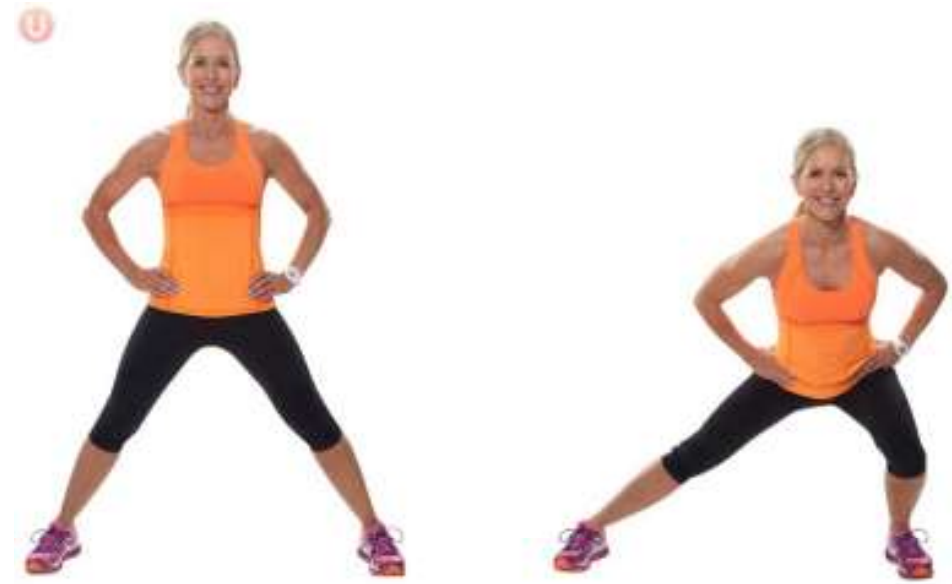

Figure 4.5: Side lunge: start and end positions [26].

pre-experimental interviews and the surveyed literature, this aspect of the movement pattern execution is easily observed by a trained coach - even when dealing with a large class.

The correct back positioning is based on the back angle, shown in Figure 4.8. The hip angle is formed by the plane of the torso and the femur. The knee angle is formed by the femur and the tibia. The back angle is formed by the plane of the torso and the floor. Although each functional exercise requires a different back angle, this aspect is also easily observable. Lumbar overextension, shown in Figure 4.9, is an execution mistake that indicates a failure to engage enough abdominal contraction to support the spine, and can lead to severe back injuries. Experienced coaches observe wrinkles that appear in the cloth of the shirt to detect if the lumbar is overextended. 


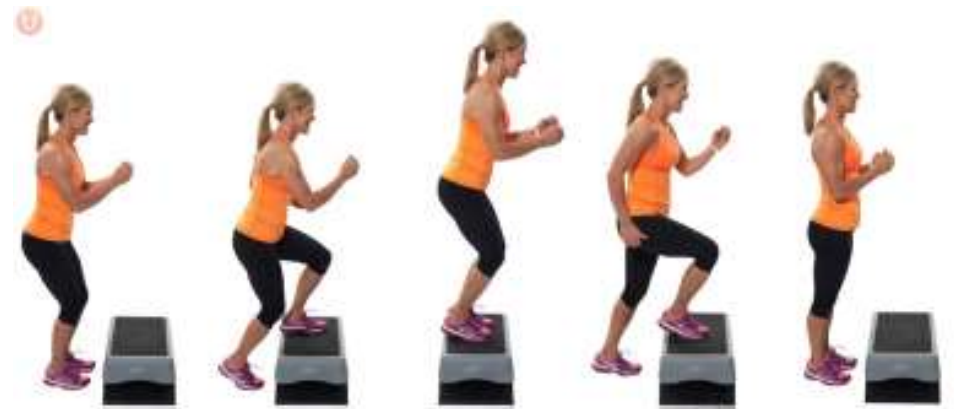

Figure 4.6: Step-up: movement pattern [26].
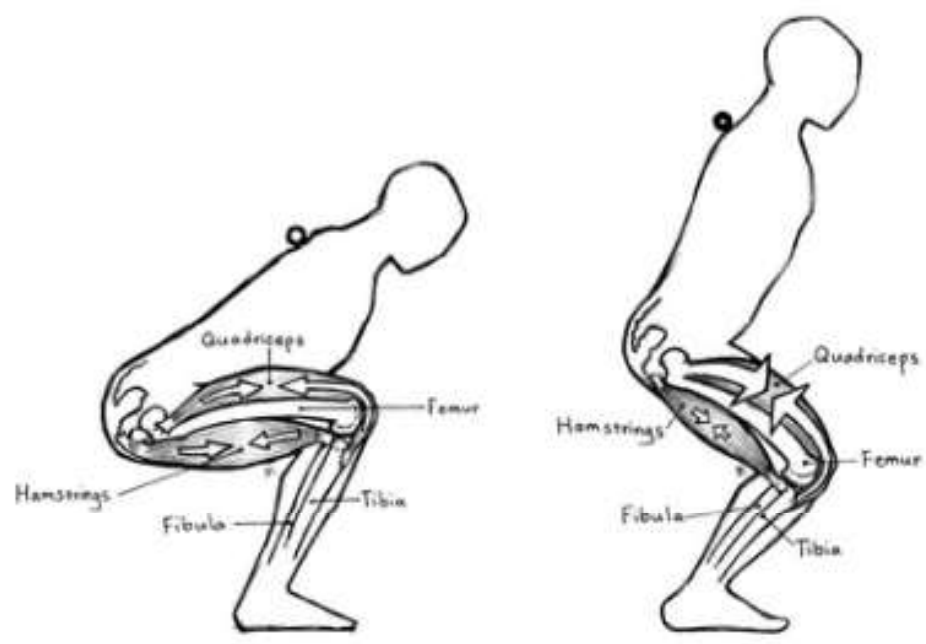

Figure 4.7: Difference between full and partial extensions [78].

The eye gaze direction is an important part in the process of performing these functional movement patterns. Looking up has detrimental effects on proper technique, interfering with (i) the correct bottom position, (ii) the hip drive out of the bottom position, and (iii) the back angle. In addition to that, it places the cervical spine in extreme overextension and can lead to severe RSI. Similar to the depth of movement and the back positioning, this aspect of the movement pattern execution is easily observed by a trained coach, regardless of class size. Based on the testimony of the certified health professionals that helped in planning of the experiment, eye gaze direction is the hardest aspect to amend in a beginner.

The correct knee positioning is directly in line with the feet, slightly out in front of the toes, so that the femurs and the feet are parallel. The exact distance and positioning is determined by the anthropometry of the individual, thus this aspect of execution is inherently difficult to observe and requires great 


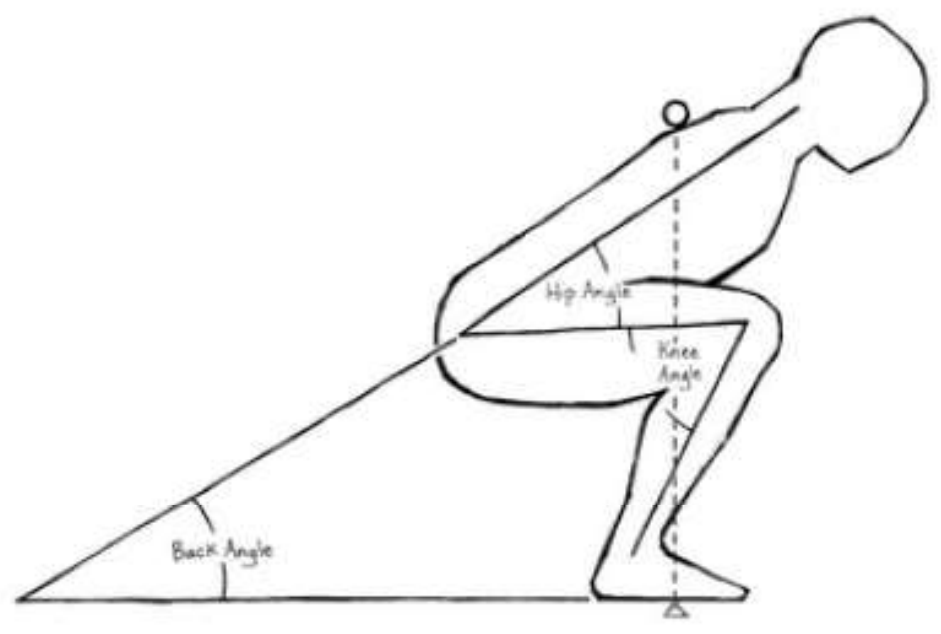

Figure 4.8: Angles of hip drive movement patterns [78].

attention to even monitor a single individual - unfeasible in larger classes. The correct foot positioning is based on three aspects: (i) the mid-foot balance point, (ii) the distance between heels and the angle of the feet, and (iii) the plantar pressure distribution. Mid-foot balance point is shown in Figure 4.10 - the musculoskeletal system maintains the tibial angle and transfers force to the sole of the foot. Extreme execution mistakes of mid-foot positioning can be noticed as the individual looses balance when performing the exercises, as shown in Figure 4.11. However, subtle errors pose a threat to the individual's health and are not observable without the aid of instruments. The correct distance between the heels and the angle of the feet, shown in Figure 4.12, can be easily observed by a trained coach, regardless of class size. The last aspect of foot positioning is the plantar pressure distribution. The musculoskeletal system is in balance when ground pressure is evenly distributed directly over the middle of the foot: the mid-foot segment - right under the arch of the foot - is the point of interaction with the ground that is farthest away from both the forward and the rearward edges of contact [78]. This aspect is not observable without the aid of instruments, and any mistake, when performed repeatedly, can lead to (i) shear on the knees, (ii) patellar RSI, and (iii) lower back injuries.

Based on this understanding of the musculoskeletal aspects responsible for the most prevalent injuries, we decided to update the platform developed in [73], [73] and [76] to collect data from (i) knee positioning, (ii) midfoot balance, and (iii) plantar pressure distribution during functional exercise training. The aim was to build the HAR classifier for the second experiment 


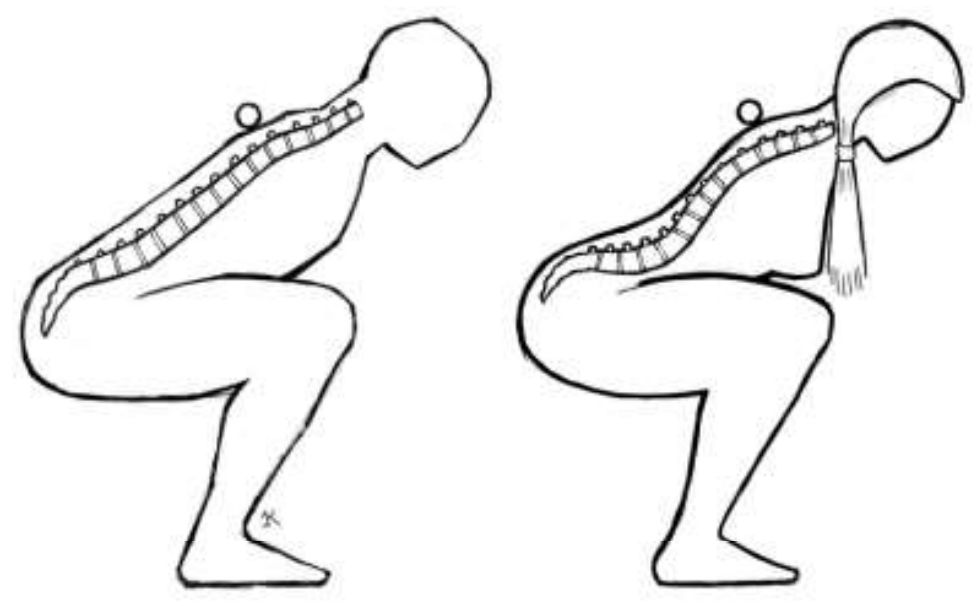

Figure 4.9: Difference between the correct back positioning, on the left, and lumbar overextension, on the right [78].

and the validation of the assessment model proposed by this work in chapter 5 .

One prevailing limitation of the surveyed the works, discussed in chapter 2 , is the employment of homogeneous participants in the experiments. This study tries to circumvent this problem with volunteers carefully selected for their diverse characteristics - age, gender, height and weight - and the participation of people with disabilities. Table 4.1 bellow summarizes participants profile information. Since the insole and shoes are US men's size 8.5, most of the selected participants fell into the 7.5 to 10.5 shoe size range. Although one female participant wore US men's size 8, the overall accuracy of her activity recognition results were similar to the other participant's.

\section{2}

\section{Conducting the Experiment}

In this section we describe in detail how the experiment was conducted. The activity model we developed for the experiment comprises 13 activities: walking straight $(2 \mathrm{~km} / \mathrm{h})$, slow jogging $(6 \mathrm{~km} / \mathrm{h})$, hopping, ascending stairs, descending stairs, standing, basic squat, sumo squat, squat hold, basic step up, front lunge, side lunge, and sitting. The experiment was conducted in 3 distinct 30-minute sessions, where participants performed a set of the planned activities monitored by one certified physical education professional. Following the advice of the physical educator, no participant performed more than 1 session per day and sessions were spaced by at least $48 \mathrm{~h}$. All sessions were performed in a personal training studio.

At each session, participants performed 6 cycles of 5-minute routines. 


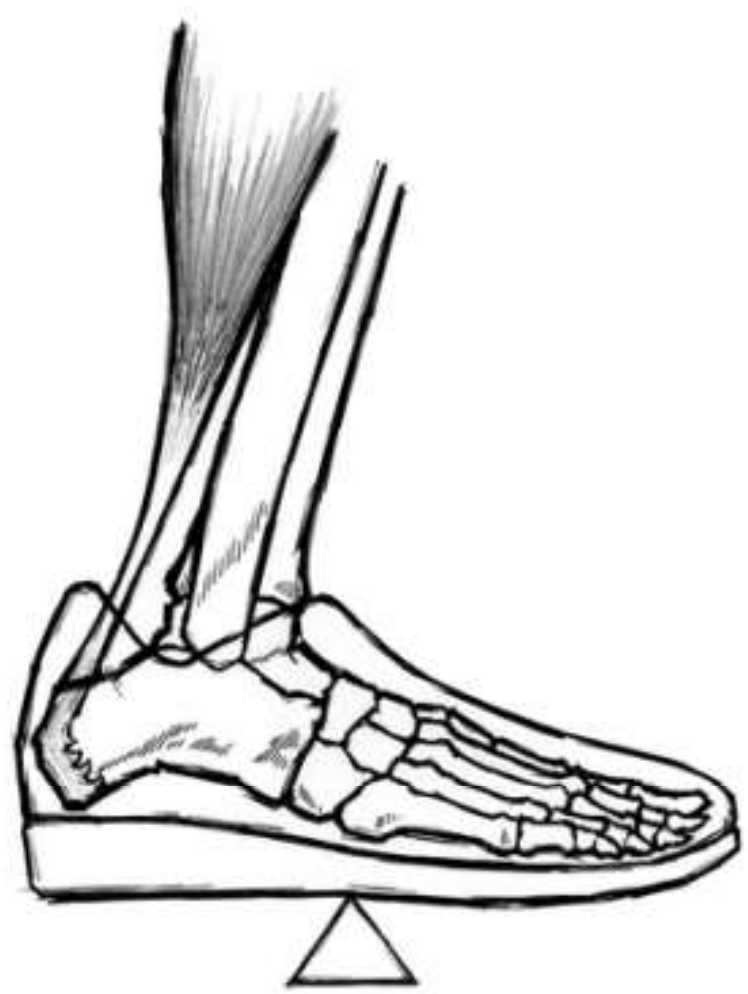

Figure 4.10: Mid-foot balance point [78].

In the first session, participants were required to interweave walking, jogging, hopping, ascending stairs, descending stairs, standing, and the execution of the Basic Step Up functional exercise. In the second session, participants were required to interweave walking, standing, sitting and the execution of the Basic Squat, Sumo Squat and Squat Hold functional exercises. In the third session, participants were required to interweave walking, standing, sitting and the execution of the Front Lunge and Side Lunge functional exercises. Subjects were free to perform the activities - the wearable prototype did not restrict in any sense their movement. All routines were developed jointly with the certified physical education professional, who provided clear instructions of how the activities were to be performed during the experiment. We focused on the time that each participant should spend performing the proposed activities, rather than the number of repetitions, as the physical conditioning of each participant varied significantly. No training weights were used, to mitigate the probability of injuries during the experiment. Table 4.2 details the physical activities performed during the data collection experiment.

We accompanied all participants during the sessions to monitor the wearable IoT device data collection and for logging any unusual occurrence. Hypoallergenic socks and foot deodorizer were used to avoid skin allergies and 


\begin{tabular}{|c|c|c|c|c|c|}
\hline Participant & Age & Gender & Height & Weight & Observations \\
\hline No.1 & 35 & Male & $1.85 \mathrm{~m}$ & $136 \mathrm{Kg}$ & $\begin{array}{l}\text { Class II Obesity \& } \\
\text { Right Lateral Meniscus Tear }\end{array}$ \\
\hline No.2 & 28 & Male & $1.74 \mathrm{~m}$ & $73 \mathrm{Kg}$ & ADHD \\
\hline No.3 & 29 & Male & $1.76 \mathrm{~m}$ & $72 \mathrm{Kg}$ & - \\
\hline No.4 & 32 & Male & $1.81 \mathrm{~m}$ & $81 \mathrm{Kg}$ & - \\
\hline No.5 & 27 & Female & $1.77 \mathrm{~m}$ & $61 \mathrm{Kg}$ & $\begin{array}{l}\text { Left Knee RSI \& } \\
\text { Left Ankle Lesion }\end{array}$ \\
\hline No.6 & 45 & Female & $1.64 \mathrm{~m}$ & $87 \mathrm{Kg}$ & Class II Obesity \\
\hline No.7 & 62 & Male & $1.93 \mathrm{~m}$ & $110 \mathrm{Kg}$ & Overweight \\
\hline No.8 & 28 & Male & $1.74 \mathrm{~m}$ & $70 \mathrm{Kg}$ & - \\
\hline No.9 & 37 & Female & $1.67 \mathrm{~m}$ & $59 \mathrm{Kg}$ & Professional Runner \\
\hline No.10 & 35 & Male & $1.54 \mathrm{~m}$ & $65 \mathrm{Kg}$ & Overweight \\
\hline No.11 & 34 & Male & $1.89 \mathrm{~m}$ & $90 \mathrm{Kg}$ & Professional Skater \\
\hline No.12 & 33 & Female & $1.61 \mathrm{~m}$ & $52 \mathrm{Kg}$ & - \\
\hline
\end{tabular}

Table 4.1: Main physical characteristics of each participant

\section{Experiment 1: Exercise routines}

Session 1

Cycle Routine

$1 \quad 1$ min walk, 2 min ascending stairs, 1 min walk, 1 min standing

21 min walk, 2 min descending stairs, 1 min walk, 1 min standing

31 min walk, 2 min Basic Step Up, 1 min walk, 1 min standing

41 min walk, 2 min Jogging, 1 min walk, 1 min standing

$5 \quad 1$ min walk, 2 min Hopping, 1 min walk, 1 min standing

61 min walk, 2 min Basic Step Up, 1 min walk, 1 min standing

Session 2

Cycle Routine

11 min walk, 2 min Basic Squat, 1 min standing, 1 min sitting

21 min walk, 2 min Sumo Squat, 1 min standing, 1 min sitting

31 min walk, 2 min Squat Hold, 1 min standing, 1 min sitting

41 min walk, 2 min Basic Squat, 1 min standing, 1 min sitting

51 min walk, 2 min Sumo Squat, 1 min standing, 1 min sitting

61 min walk, 2 min Squat Hold, 1 min standing, 1 min sitting

Session 3

Cycle Routine

11 min walk, 2 min Front Lunge, 1 min standing, 1 min sitting

21 min walk, 2 min Side Lunge, 1 min standing, 1 min sitting

31 min walk, 2 min Front Lunge, 1 min standing, 1 min sitting

41 min walk, 2 min Side Lunge, 1 min standing, 1 min sitting

$5 \quad 1$ min walk, 2 min Front Lunge, 1 min standing, 1 min sitting

$61 \mathrm{~min}$ walk, $2 \mathrm{~min}$ Side Lunge, $1 \mathrm{~min}$ standing, $1 \mathrm{~min}$ sitting

Table 4.2: Data collection experiment exercise routines. 

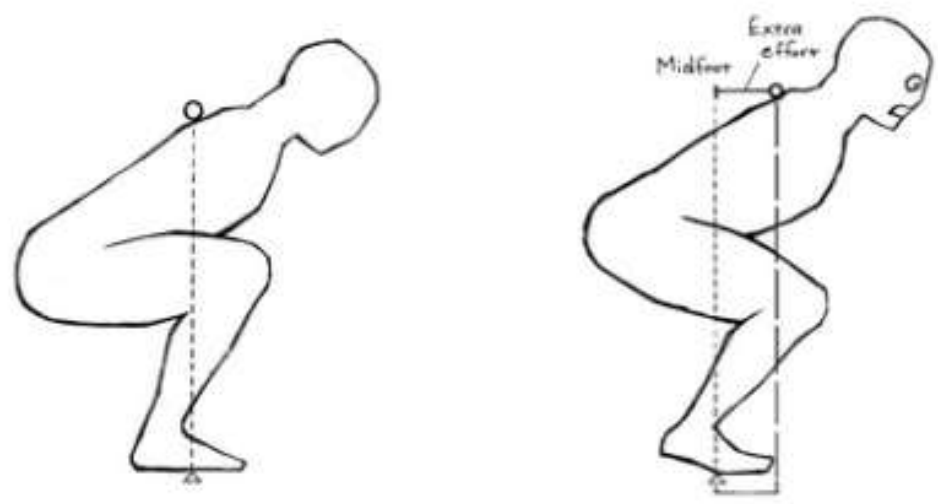

Figure 4.11: Unbalance due to mid-foot positioning mistakes [78].
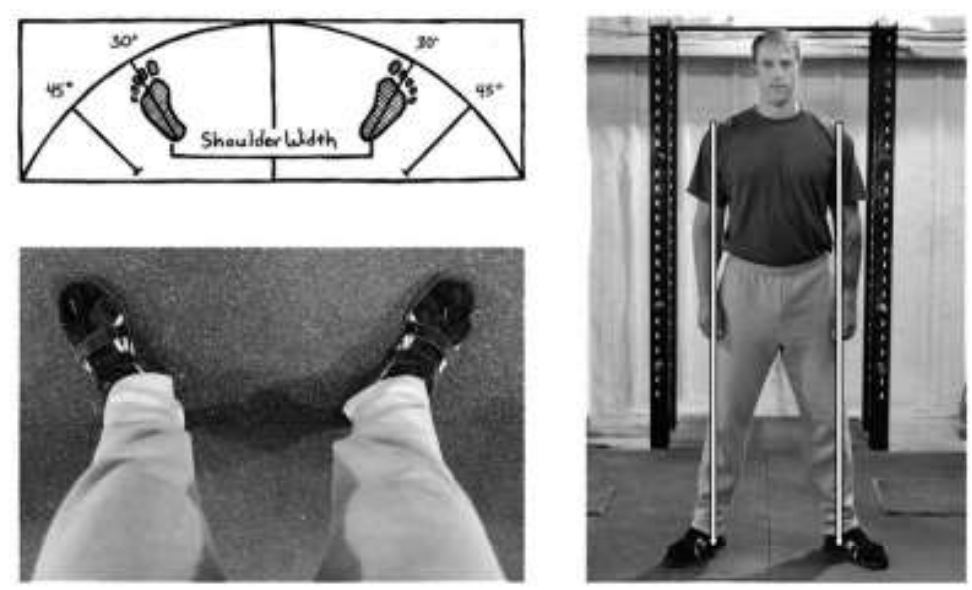

Figure 4.12: Correct heel positioning and feet angle [78].

prevent any discomfort. In total, 18 hours of activity data were collected 90 minutes of feet and knee posture and movement data from each volunteer. The number of subjects in our study is not that different from the surveyed works others, considering the median of 6 found in the literature. However, the number of samples in our study - over 1.2 million - is significantly higher than the median number of samples - around 50,000 - found on the surveyed works.

\section{3}

\section{Data Acquisition}

During the experiment, a stream of unprocessed sensor signals - built from the combination of the insole's sensors and the knee's sensors - is stored in the microcontroller in JSON format. This raw data combines two accelerometers, two gyroscopes, two magnetometers, six FSR sensors, altitude, pressure, and two range finder sensor signals, resulting in 28-feature set entries 
to the dataset. Following the recommendations of [38], we used a $10 \mathrm{~Hz}$ sampling rate to recognize between similar activities and subtle variations in execution style. This study investigated the effects of sample size and time window lengths in HAR, using an activity model with eight activities: standing, walking, sitting, climbing stairs up, climbing stairs down, lying, turning and running - an activity model similar to the one used by this work. Also similar to this work, the study in [38] employs decision trees algorithms and tenfold cross-validation - the same methods used to build the HAR classifier presented in this chapter. The JSON formatted data was periodically sent to the application server in small packages of $200 \mathrm{~KB}$ to reduce energy and data usage.

\section{4}

\section{Data Processing, Feature Extraction and Selection}

A data processing pipeline of two steps, similar to the one proposed in [73] and [76], was employed. No experiment data was discarded, given that the prototype starts collecting feet movement and posture information immediately after it is powered. First, the dataset was labelled for supervised learning, and activity class information was appended to each entry according to the activity performed in the experiment. All sensor data was normalized, to make the scales between participants equivalent for the model building.

In the feature extraction stage, we used descriptive statistics - standard deviation, variance, minimum, maximum and average values - to generate derived features from each of the 28 original features:

- Six FSR sensor readings;

- Six gyroscope axis data;

- Six magnetometer axis data;

- Six accelerometer axis data;

- Altitude reading;

- Pressure reading; and,

- Two range finder sensors reading.

Moreover, as employed by some of the surveyed works and the research previously conducted, (i) the cumulative difference between samples for each feature and (ii) the Euler angles of pitch, roll and yaw were used to generate additional derived features, for a total of over 200 features for selection.

As in [73] and [74], we employed Hall's algorithm [37] based on correlation for feature selection, using its default "Best Fit" backtracking greedy strategy 
configuration. In total, 22 features were selected by this method. As in [46], [75] and [74], the range finder sensors were successfully employed and improved the average accuracy of our results. Unlike the classifier proposed in [73], the altimeter was not employed by the classifier. Using the features selected by Hall's algorithm, our HAR classifier was built based on:

- Four FSR sensor readings;

- Two axes of the foot gyroscope;

- Three axes of the knee gyroscope;

- Two axes of the foot magnetometer;

- Two axes of the knee magnetometer;

- One axes of the foot accelerometer;

- Two axes of the knee accelerometer;

- Two Euler angles of the foot; and,

- Maximum and minimum of the range finder sensors.

\section{5}

\section{Classification and Validation}

We experimented different strategies to build the HAR classifier model, such as Bagged Decision Trees, Classification and Regression Trees, k-Nearest Neighbors, Naive Bayes, and Support Vector Machines. Following the results found in the research conducted previously in [73, 75, 76], the Random Forest Algorithm achieved better results - with an overall classification accuracy of about $87 \%$ for activity recognition - a drop of $6.26 \%$ in the overall accuracy when compared to the first activity model proposed in [73]. Random Forests, as ensemble methods, tend to decrease variance and bias and improve predictions. We set the hyper parameter of the Number of Trees to 128, restricted the hyper parameter Depth to 15 to avoid overfitting and employed all selected features. This configuration is expected to efficiently produce a highly accurate classifier, even on large databases with hundreds of features. To validate the model, we applied the Leave-one-out Cross Validation in attempt to increase the robustness of the model - since this method guarantees that both training and test splits does not share any example data. For full disclosure, the individual validation results for each of the 12 participants are shown on Table 4.3. Although the achieved results are similar to the top results found in the literature for complex activity models, as discussed in chapter 2, since each research employed a different dataset, activity model, algorithm customization and validation methods, it is not possible to compare the results. 


\begin{tabular}{lll}
\multicolumn{3}{l}{ Classification and validation results } \\
Participant & Precision & Recall \\
\hline No.1 & 81.1 & 59.9 \\
No.2 & 79.3 & 54.2 \\
No.3 & 94.3 & 72.2 \\
No.4 & 92.2 & 74.6 \\
No.5 & 84.2 & 68.1 \\
No.6 & 82.7 & 59.4 \\
No.7 & 87.5 & 68.8 \\
No.8 & 90.1 & 79.0 \\
No.9 & 96.4 & 80.4 \\
No.10 & 74.7 & 51.4 \\
No.11 & 89.5 & 64.3 \\
No.12 & 92.5 & 70.5
\end{tabular}

Table 4.3: Results per participant

For the time window sizes to build the classifier, we evaluated different alternatives and decided to use a 3-seconds window based on our model validation results. Although [8] recommends window sizes within the $0.25 \mathrm{~s}$ - 0.50s range for single activity recognition, our activity model consists of complex, compound multi-jointed exercises, and smaller window sizes did not achieve good overall classification accuracy. 


\section{Second Experiment and Methodology Evaluation}

In this chapter, we describe the stages followed to validate the proposed pattern recognition assessment model: performing a second experiment and conducting a semi-structured interview to evaluate usability and user experience.

\section{1}

\section{The Second Experiment}

During the second experiment we employed 9 of the 12 original first experiment volunteers - three of them were not available at the time it was conducted. Table 5.1 summarizes Experiment 2 participants information.

The goal of the second experiment was to validate the feasibility of the HAR classifier for and its capability to assess if the movement pattern of a particular functional training exercise was performed correctly by the user. To achieve that goal, we used the same wearable IoT device employed to build the model with two modifications: (i) we added a SparkFun Haptic Motor Driver with the DRV2605L by Texas Instruments, together with a vibration motor, and (ii) we altered the server code to send a return code that indicated if the activity was performed correctly. As discussed in chapter 2, audible feedback is inadequate for CrossFit ${ }^{\mathrm{TM}}$ classes based on the typical high sound employed by coaches during classes. After the conclusion of the second experiment, we

\begin{tabular}{llllll}
\multicolumn{6}{l}{ Experiment 2: participant profile summary } \\
Participant & Age & Gender & Height & Weight & Observations \\
\hline No.1 & 35 & Male & $1.85 \mathrm{~m}$ & $136 \mathrm{Kg}$ & Class II Obesity \& \\
& & & & & Right Lateral Meniscus Tear \\
No.2 & 28 & Male & $1.74 \mathrm{~m}$ & $73 \mathrm{Kg}$ & ADHD \\
No.3 & 29 & Male & $1.76 \mathrm{~m}$ & $72 \mathrm{Kg}$ & - \\
No.6 & 45 & Female & $1.64 \mathrm{~m}$ & $87 \mathrm{Kg}$ & Class II Obesity \\
No.7 & 62 & Male & $1.93 \mathrm{~m}$ & $110 \mathrm{Kg}$ & Overweight \\
No.8 & 28 & Male & $1.74 \mathrm{~m}$ & $70 \mathrm{Kg}$ & - \\
No.9 & 37 & Female & $1.67 \mathrm{~m}$ & $59 \mathrm{Kg}$ & Professional Runner \\
No.10 & 35 & Male & $1.54 \mathrm{~m}$ & $65 \mathrm{Kg}$ & Overweight \\
No.12 & 33 & Female & $1.61 \mathrm{~m}$ & $52 \mathrm{Kg}$ & -
\end{tabular}

Table 5.1: Main physical characteristics of each participant 


\section{Experiment 2: Exercise routines}

Session 1

Cycle Routine

11 min walking, 2 min Basic Step Up, 1 min standing, 1 min sitting

21 min walking, 2 min Front Lunge, 1 min standing, 1 min sitting

31 min walking, 2 min Side Lunge, 1 min standing, 1 min sitting

41 min walking, 2 min Basic Squat, 1 min standing, 1 min sitting

51 min walking, 2 min Sumo Squat, 1 min standing, 1 min sitting

61 min walking, 2 min Squat Hold, 1 min standing, 1 min sitting

Table 5.2: Model validation experiment exercise routines.

replaced the Haptic Motor Driver and the vibration motor for a light-emitting diode (LED) for demonstration purposes.

The second experiment was conducted in a 30-minute session, where participants performed a set of the planned activities monitored by the same certified health professionals of the first experiment. This session was performed at the same personal training studio used for the first experiment. No data was collected during the second experiment. At the session, the participants performed 6 cycles of 5-minute routines. The routines consisted of all 13 activities of our activity model, and were also developed jointly with the certified physical education professionals. No instructions of how the activities were to be performed were provided during the experiment, although every question posed by the participants was answered. A few participants that were performing correctly every activity of the proposed routines were asked to alter the movement pattern to replicate the most common execution errors found during a functional exercise practice. This was done for no more than 3 repetitions for each activity - since the repetitions were performed without extra weights and on foam mat floor tiles, the physical educator ensured that no harm would befall those participants. Once more, we focused on the time that each participant should spend performing the proposed activities, rather than the number of repetitions, as the physical conditioning of each participant varied significantly. Table 5.2 details the performed activities:

During each cycle, participants performed only one of the 6 specific functional exercises of our activity model. We configured the server application to assess only the matching functional exercise each cycle, sending a return code of 0 if the functional exercise - basic step up, basic squat, sumo squat, squat hold, front lunge, and side lunge - movement pattern was performed correctly and 1 otherwise. A return code of 1 activated the vibration motor, warning the user of the movement pattern performed. We asked one health professional to take notes during the sessions, registering each time a functional 


\begin{tabular}{|c|c|c|c|}
\hline $\begin{array}{l}\text { Assessmen } \\
\text { Participant }\end{array}$ & $\begin{array}{l}\text { model result } \\
\text { Basic step up }\end{array}$ & $\begin{array}{l}\text { Step up a } \\
\text { Front lunge }\end{array}$ & $\begin{array}{l}\text { ad lunges } \\
\text { Side lunge }\end{array}$ \\
\hline No.1 & $28 / 2$ & $6 / 4$ & $5 / 5$ \\
\hline No.2 & $24 / 6$ & $6 / 6$ & $4 / 8$ \\
\hline No.3 & $28 / 2$ & $4 / 4$ & $3 / 5$ \\
\hline No.6 & $22 / 3$ & $6 / 2$ & $6 / 2$ \\
\hline No.7 & $12 / 3$ & $4 / 2$ & $4 / 2$ \\
\hline No.8 & $18 / 2$ & $4 / 2$ & $3 / 3$ \\
\hline No.9 & $24 / 1$ & $8 / 0$ & $7 / 1$ \\
\hline No.10 & $10 / 2$ & $5 / 1$ & $1 / 3$ \\
\hline No.12 & $19 / 1$ & $10 / 2$ & $10 / 2$ \\
\hline Total: & $185 / 22$ & $53 / 23$ & $43 / 31$ \\
\hline
\end{tabular}

Table 5.3: Assessment model evaluation 1

Assessment model results: Squats

\begin{tabular}{llll} 
Participant & Basic squat & Sumo squat & Squat hold \\
\hline No.1 & $9 / 1$ & $5 / 1$ & $3 / 1$ \\
No.2 & $6 / 4$ & $3 / 2$ & $4 / 1$ \\
No.3 & $4 / 2$ & $4 / 1$ & $3 / 2$ \\
No.6 & $6 / 2$ & $5 / 1$ & $3 / 1$ \\
No.7 & $5 / 1$ & $3 / 1$ & $1 / 3$ \\
No.8 & $3 / 3$ & $4 / 2$ & $4 / 2$ \\
No.9 & $7 / 1$ & $5 / 3$ & $6 / 2$ \\
No.10 & $4 / 2$ & $5 / 1$ & $2 / 2$ \\
No.12 & $9 / 1$ & $9 / 1$ & $9 / 1$ \\
\hline Total: & $53 / 17$ & $43 / 13$ & $35 / 15$
\end{tabular}

Table 5.4: Assessment model evaluation 2

exercise movement was performed poorly. The other movements were not analysed during the sessions, since (i) walking was added for warming-up and (ii) standing and sitting were added for resting purposes. The definition of a correctly performed movement, during the second experiment, was based on the health professional's observations log and post-session interview. Tables 5.3 and 5.4 details the performed activities. Each column represents a functional exercise performed during the second experiment. The numbers on the left indicate the number of correct executions and the numbers on the right indicate the number of execution errors.

In total, participants executed 421 correct movement patterns and made 121 execution mistakes, as observed by the health professional. Of the 121 mistakes, 81 were recognized by the assessment model, accounting for a recall of $\approx 66.9 \%$. In the opinion of the certified health professionals, this result is adequate for deployment, since the goal is the prevention of repeated mistakes, not the detection of single execution mistakes. Of the 421 correctly executed 
movement patterns, 12 were recognized by the assessment model as execution mistakes. This high-enough precision, of $\approx 87.1 \%$, is a particularly important result, since misplaced feedback is detrimental to the athlete's understanding of the movement pattern execution.

\subsection{1}

\section{The Interview}

After the second experiment, we performed a semi-structured interview to evaluate usability and user experience. We interviewed all participants from the second experiment after their sessions, and the certified health professionals after the study was concluded. We documented all the information provided by the participants and certified health professionals. In addition to that, we interviewed one participant of the first experiment that did not participate in the second experiment. The following questions were asked to the participants:

- Did the device or the haptic feedback hinder in any way the execution of the activities?

- Do you perceive the haptic feedback as a help to perform the proposed activities?

- Do you think that the exercise execution assessment platform proposed is necessary or adequate for your regular physical training program?

Overall, the participants did not consider that the device hindered the execution of the activities, since it is lightweight and the flexible cable connecting the knee band and the foot external protective case was well positioned and of adequate length. Only one participant, below the average height of the participants, reported the cable as a minor nuisance during the sessions. However, 3 of the 10 respondents reported that they were over overzealous with their movements to prevent any damage to the wearable IoT device, so in their perception the device hindered their focus during the exercises execution. Of those 3 respondents, 2 reported that the impaired focus was the probable cause of some execution mistakes. The participants knew that there was only one prototype available and that the research group did not receive any grant to fund the research. Considering that the IoT wearable device used in the experiment is a prototype that could be improved, with more resources, with the removal of the cable and the development of a more robust circuitry and casing, and the feedback provided, we may assume that the proposed platform does not hinder the execution of the activities that comprise the activity model investigated in this work. The haptic feedback was perceived as a help factor to perform exercises more correctly by 8 of the 9 respondents, 
although 6 of those respondents pointed out that a human feedback is more helpful since it enables them to understand the reason why an execution was performed incorrectly. It is important to observe that the only respondent who did not think the haptic feedback is a help factor to perform exercises more correctly is a professional half marathon and $5 \mathrm{~K}$ runner, with extensive physical education experience. Although only a single answer, it suggests that the feedback is more useful for beginner and intermediate athletes. All seven physically active participants train without the individual guidance of a physical educator by cause of monetary costs or complex schedule. They and agreed that (i) an exercise execution assessment platform is necessary in the absence of a coach or spotter and that (ii) the proposed model and the type of device employed - after adjustments necessary for production, as discussed above - are adequate for their training regimen. Some respondents reported that the study helped them realize the importance of coaching during training for their long-term safety, and that they would consider buying products or subscribing to services that provide exercise execution feedback.

To the certified health professionals, we asked the following questions:

- Do you perceive the IoT wearable device as an impediment to the execution of the activities of the experiment?

- Do you perceive the haptic feedback as a help to perform the proposed activities?

- Do you think that the exercise execution assessment platform proposed is necessary or adequate for your regular practice as a physical educator?

Both respondents did not consider that the device an impediment to the execution of the activities, however, they agree that the cable is not adequate to most of the physical practice environments, especially the gymnasium. Regarding the haptic feedback both reported that personalized real-time feedback is key to the safe practice of physical activities. One of the professionals stated that they were amazed by the use of such technology in their field, and did not provide any further information. The other professional believes that haptic feedback on the feet is not suitable for (i) most of the physical activities, such as running, jumping or climbing, where there is already a sensory overload on the body part or where the athlete is barefooted and (ii) that haptic feedback on any part of the body is not suitable for some physical activities, such as swimming or grappling martial arts, where the athlete will not be able to focus his tactile perception on the device. However, he considers the haptic feedback extremely helpful for a niche of complex exercises, such as functional training and weightlifting. In addition to that, he suggested to conduct a similar study 
to investigate precision-based Olympic Sports, such as archery and shooting. Both professionals agreed that (i) a typical functional exercise class equipped with a physical assessment platform could be monitored by only one coach, able to make better use of his time instead of monitoring every aspect of the execution, (ii) that a physical assessment platform capable of monitoring aspects of the execution not easily assessed by an observational approach, such as plantar pressure distribution, greatly enhances their performance as coaches, and (iii) despite the real-life accuracy below $70 \%$ the model is adequate for deployment, since the key factor regarding RSI prevention is the detection of repeated mistakes, not the detection of a single poor execution. One professional also stated that, although non-ideal, the proposed platform could enable them to coach a geographically distributed class - with the aid of cameras and monitors - or a private client on a trip.

Finally, the respondents stated that constant feedback is not the best strategy to amend a flawed execution, and any feedback provided should respect the individual's (i) tolerance for criticism and (ii) capacity to progress in a single session. Their belief is that a post-execution or real-time private report, to the coaches, could allow for a more effective approach than one of real-time feedback. 


\section{6 Conclusion}

This work proposes a platform for physical evaluation of functional exercise activities with real-time feedback to help reduce injury risk. We conducted two experiments: (i) the first with 12 volunteers, to build a HAR classifier of a 13-classes activity model based on foot and knee movement and posture information and (ii) the second with 9 volunteers, to validate the physical evaluation model and investigate usability issues of the proposed wearable IoT device. The platform was considered helpful by the experiment participants and the supervising physical educators.

The main contributions are:

- A public domain dataset with about 1 million samples and 12 activity classes, available in [73];

- A platform and a HAR model that can be used to assess the quality of execution of different lower limb functional exercises; and,

- A wearable IoT device blueprint with a comprehensive and novel sensor fusion selection that can be used for lower limb HAR.

The platform and HAR model proposed in this work were first developed for the recognition of simple activities, based on an activity model with 6 activities - walking straight, walking slope up, walking slope down, ascending stairs, descending stairs and sitting -, and published in [73]. An investigation of the sensor type relevance for HAR followed the initial research, employing the same platform and HAR model, and published in [74]. The sensor mix and wearable IoT device were updated to assess physical activity engagement, with an activity model comprising 10 activities - walking straight $(2 \mathrm{~km} / \mathrm{h})$, walking slope up $(2 \mathrm{~km} / \mathrm{h})$, walking slope down $(2 \mathrm{~km} / \mathrm{h})$, slow jogging $(6 \mathrm{~km} / \mathrm{h})$, slow jogging slope up $(6 \mathrm{~km} / \mathrm{h})$, slow jogging slope down $(6 \mathrm{~km} / \mathrm{h})$, hopping, ascending stairs, descending stairs, and sitting. Finally, with the addition of the knee sensors, the wearable IoT device and HAR model were employed for the assessment of quality of functional exercises to mitigate the probabilities of injuries. The conducted research is published in [76] and is the foundation this work. 
Our assessment model achieved a precision of $\approx 87.1 \%$, and recall of $\approx 66.9 \%$, when determining if a functional exercise was performed correctly. This result, as discussed in chapter 5, was adequate for the proposed goal. However, if necessary, it is possible to improve the HAR Classifier for the activity model used in this work by (i) deploying sensors on the lower back, (ii) deploying different sensor types on the knees, and (iii) conducting another experiment with a greater number of diverse participants. In addition to that, a new model employing participant's features, such as age, height and weight, could be devised to attempt to improve overall accuracy. However, based on the number of participants, measures to avoid overfitting should be thoroughly considered.

Although many studies focus on the recognition of activities, the assessment of quality of execution is still a challenge of the HAR research field. Quality is a subjective characteristic, and some of its agreed aspects may not be easily observed - even by sensors and video recording devices. In this study, the definition of exercise execution quality was derived from an agreement between the two certified health professionals that participated in the planning and conduction of the experiments - the developed HAR classifier is their opinion embedded in a statistical model. Although this work was structured to facilitate replication by other researchers, the achieved classification and validation results of this model will always be dependent upon the observations of the selected certified health professionals. Concerning the proposed model, one limitation of this work is the binary approach to the assessment of an individual's movement pattern, where a movement patterns is defined as either correct or incorrect, as apposed to an approach where an incorrect movement pattern is graded according to the severity of the mistake.

It is possible that, in the context of exercise execution quality assessment, a more effective approach could be developed based on recommender systems. Similar to the cold start problem [52], an initial HAR classifier, based on the characteristics of the user, could be offered for a first time user. Then, after each use, the collected data could be employed to continuously tailor the HAR classifier to the specific needs of its user. Considering that approach, a scenario worth investigating is (i) the long-term monitoring of a health professional, (ii) supported by the platform proposed in this work, with (iii) a periodic revision of the custom-made HAR classifier with newly-collected data and the individual's health condition. Another challenge worth investigating is the deployment of sensors and their impact on the accuracy of HAR classifiers for specific activity models. The optimal quantity of sensors and their positioning, particularly the FSRs, remain unanswered in the surveyed 
literature. Finally, a bigger challenge lies in the use of electromyography to enhance the assessment of execution mistakes and enable the monitoring of complex movement patterns.

We hypothesize that, although not reported by the participants, the performed physical exercise routines in the second experiment were excessively demanding. The small volume of repetitions of the basic squat, sumo squat and squat hold exercises, performed last, suggest that the participants could have been fatigued during their execution. In addition to that, the exhaustion level of the participants during the execution of the last exercises could have an influence on the execution mistakes. Since the basic squat, sumo squat and squat hold exercises are considered, by the health professionals, to be more complex than the basic step up, front lunge and side lunge exercises, the achieved results are not conclusive regarding this observation.

Currently, we plan to evolve the proposed wearable IoT device prototype aiming at the capability to build HAR classifiers for a broader activity model, comprising full-body movement patterns. One alternative is the use of a BSN and the deployment of sensors on the lower back, shoulders and head, to collect data from those body parts. Our goal is to use the evolved wearable IoT prototype to assess the quality of execution of movement patterns in collective sports, such as basketball or soccer, or martial arts based on hand and foot strikes, such as boxing and kickboxing. 


\section{Bibliography}

[1] Agha, M., Agha, R.: The rising prevalence of obesity: part a: impact on public health. In: International journal of surgery. Oncology. pp. 2-17 (2017)

[2] Ahmadi, A.e.a.: Automatic activity classification and movement assessment during a sports training session using wearable inertial sensors. In: 11th International Conference on Wearable and Implantable Body Sensor Networks (2014)

[3] Anderson, R.e.a.: Accelerometry-based feedback - can it improve movement consistency and performance in rowing? In: Sports Biomechanics Vol.4 (2) 179-195 (2005)

[4] Au, L.K., Wu, W.H., Batalin, M.A., Mclntire, D.H., Kaiser, W.J.: Microleap: Energy-aware wireless sensor platform for biomedical sensing applications. In: Biomedical Circuits and Systems Conference. BIOCAS. pp. 158-162. IEEE (2007)

[5] Auvinet, B.e.a.: Runner's stride analysis: comparison of kinematic and kinetic analyses under field conditions. In: Science \& Sports; 17 : 92-4. pp. 92-94 (2002)

[6] Baca, A., Kornfeind, P.: Rapid feedback systems for elite sports training. In: IEEE CS and IEEE ComSoc (2006)

[7] Bachlin, M.e.a.: Swimmaster: A wearable assistant for swimmer. In: UbiComp (2009)

[8] Banos, O., Galvez, J.M., Damas, M., Pomares, H., Rojas, I.: Window size impact in human activity recognition. Sensors 14(4), 6474-6499 (2014)

[9] Barfield J., A.A.: Effect of crossfit on health-related physical fitness: A pilot study. In: Journal of Sport and Human Performance (2014)

[10] Bergeron, M.e.a.: Consensus paper on extreme conditioning programs in military personnel. In: Consortium for Health and Military Performance and American College of Sports Medicine (2011) 
[11] Bevans, K., Fitzpatrick, L., Sanchez, B.: Physical education resources, class management, and student physical activity levels: A structure-processoutcome approach to evaluating physical education effectiveness. In: Journal of School Health. pp. 573-580. Wiley (2010)

[12] Carlson, S.A., Fulton, J.E., Lee, S.M., Maynard, L.M., Brown, D.R., Kohl, Harold W III, P., Dietz, W.H.: Physical education and academic achievement in elementary school: Data from the early childhood longitudinal study. In: American Journal of Public Health. pp. 721-727. American Public Health Association (2008)

[13] Crea, S., De Rossi, S., Donati, M., Reberšek, P., Novak, D., Vitiello, N., Lenzi, T., Podobnik, J., Munih, M., Carrozza, M.: Development of gait segmentation methods for wearable foot pressure sensors. In: Engineering in Medicine and Biology Society (EMBC). pp. 5018-5021. IEEE (2012)

[14] Davey, N.e.a.: An accelerometer based system for elite athlete swimming performance analysis. In: Smart Structures, Devices, and Systems II, Proceedings of SPIE Vol. 5649 (2005)

[15] De Ridder, E. M., V.O.J.O.V.A.V.G.G., Danneels, L.A.: Posterior muscle chain activity during various extension exercises: an observational study. BMC Musculoskelet Disorders 14, 100 (2013)

[16] De Santis, A., Gambi, E., Montanini, L., Raffaeli, L., Spinsante, S., Rascioni, G.: A simple object for elderly vitality monitoring: The smart insole. In: Mechatronic and Embedded Systems and Applications (MESA), ASME. pp. 1-6. IEEE (2014)

[17] Dominski, F.e.a.: Injury profile in crossfit practitioners: systematic review. In: Fisioterapia e Pesquisa. pp. 229-239 (2018)

[18] Doppler, J., Holl, G., Ferscha, A., Franz, M., Klein, C., dos Santos Rocha, M., Zeidler, A.: Variability in foot-worn sensor placement for activity recognition. In: Wearable Computers, 2009. ISWC'09. International Symposium on. pp. 143-144. IEEE (2009)

[19] Drobny, D., W.M., Borchers, J.: Saltate!: a sensor-based system to support dance beginners. In: CHI'09 Extended Abstracts on Human Factors in Computing Systems. pp. 3943-3948. ACM (2009)

[20] Eather N., M.P., Lubans, D.: Improving health-related fitness in adolescents: The crossfit teens randomised controlled trial. In: Journal of Sports. pp. 209$223(2016)$ 
[21] Edgar, S.R., Swyka, T., Fulk, G., Sazonov, E.S.: Wearable shoe-based device for rehabilitation of stroke patients. In: Engineering in Medicine and Biology Society (EMBC), 2010 Annual International Conference of the IEEE. pp. 3772-3775. IEEE (2010)

[22] El Achkar, C.M., Massé, F., Arami, A., Aminian, K.: Physical activity recognition via minimal in-shoes force sensor configuration. In: Pervasive Computing Technologies for Healthcare. pp. 256-259. ICST (2013)

[23] Ermes, M.e.a.: Detection of daily activities and sports with wearable sensors in controlled and uncontrolled conditions. In: IEEE Transactions on Information Technology in Biomedicine, VOL. 12, NO. 1, January (2008)

[24] FCl: Amphenol clincher connector datasheet. https://www.sparkfun.com/products/14194 (2017), retrieved: July, 2019

[25] Freburger, J.e.a.: The rising prevalence of chronic low back pain. In: Arch Intern Med. (2009)

[26] Freytag, C.: Get healthy u. https://gethealthyu.com/ (2019), retrieved: July, 2019

[27] Fukahori, K., Sakamoto, D., Igarashi, T.: Exploring subtle foot plantarbased gestures with sock-placed pressure sensors. In: Proceedings of the 33rd Annual ACM Conference on Human Factors in Computing Systems. pp. 3019-3028. ACM (2015)

[28] Fulk, G.D., Edgar, S.R., Bierwirth, R., Hart, P., Lopez-Meyer, P., Sazonov, E.: Identifying activity levels and steps in people with stroke using a novel shoe-based sensor. Journal of Neurologic Physical Therapy 36(2), 100 (2012)

[29] Fulk, G.D., Sazonov, E.: Using sensors to measure activity in people with stroke. Topics in stroke rehabilitation 18(6), 746-757 (2011)

[30] G., G.: Understanding crossfit. In: The CrossFit Journal. pp. 1-2 (2007)

[31] Ghobadi, M., Esfahani, E.T.: Foot-mounted inertial measurement unit for activity classification. In: Engineering in Medicine and Biology Society, EMBC. pp. 6294-6297. IEEE (2014)

[32] Haddock, C.e.a.: The benefits of high intensity functional training (hift) fitness programs for military personnel. In: Military Medicine. pp. 1508-1514 (November 2016) 
[33] Hadeed M.J., Kuehl K.S., E.D.S.A.: Exertional rhabdomyolysis after crossfit exercise program. In: Medicinal Science, Sports Exercises. pp. 224-225 (2011)

[34] Haescher, M., Matthies, D.J.C., Bieber, G., Urban, B.: Capwalk: A capacitive recognition of walking-based activities as a wearable assistive technology. In: Proceedings of the 8th ACM International Conference on PErvasive Technologies Related to Assistive Environments. pp. 35:1-35:8. PETRA '15, ACM, New York, NY, USA (2015). https://doi.org/10.1145/2769493.2769500, http://doi. acm .org/10.1145/2769493.2769500

[35] Haescher, M., Matthies, D.J., Bieber, G., Urban, B.: Capwalk: A capacitive recognition of walking-based activities as a wearable assistive technology. In: International Conference on PErvasive Technologies Related to Assistive Environments. p. 35. ACM (2015)

[36] Hak, P.T., H.E., Hickey, B.: The nature and prevalence of injury during crossfit training. In: Journal of Strength and Conditioning Research. pp. 482-485 (2013)

[37] Hall, M.: Correlation-based feature subset selection for machine learning. Thesis submitted in partial fulfillment of the requirements of the degree of Doctor of Philosophy at the University of Waikato (1998)

[38] Harasimowicz, A., Dziubich, T., Brzeski, A.: Accelerometer-based human activity recognition and the impact of the sample size. Advances in Neural Networks, Fuzzy Systems and Artificial Intelligence pp. 130-135 (2014)

[39] Hegde, N., Bries, M., Swibas, T., Melanson, E., Sazonov, E.: Automatic recognition of activities of daily living utilizing insole based and wrist worn wearable sensors. IEEE journal of biomedical and health informatics (2017)

[40] Hegde, N., Melanson, E., Sazonov, E.: Development of a real time activity monitoring android application utilizing smartstep. In: Engineering in Medicine and Biology Society (EMBC), 2016 IEEE 38th Annual International Conference of the. pp. 1886-1889. IEEE (2016)

[41] Hegde, N., Sazonov, E.: Smartstep: A fully integrated, low-power insole monitor. Electronics 3(2), 381-397 (2014)

[42] Holleczek, T., Ruegg, A., Harms, H., Troster, G.: Textile pressure sensors for sports applications 9th ieee sensors conf. Kona, HI (2010) 
[43] Hruby A., H.F.: The epidemiology of obesity: A big picture. In: Pharmacoeconomics. pp. 673-689 (2015)

[44] IBGE: Síntese de indicadores 2015. In: Pesquisa Nacional por Amostra de Domicílios (PNAD) (2010)

[45] Interlink-Electronics: $\quad$ Fsr $402 \quad$ datasheet. https://www.digikey.com/en/product-highlight/i/interlink/fsr-402-roundforce-sensing-resistor (2011), retrieved: July, 2019

[46] Jiang, X., Chen, Y., Liu, J., Hayes, G.R., Hu, L., Shen, J.: Air: recognizing activity through ir-based distance sensing on feet. In: International Joint Conference on Pervasive and Ubiquitous Computing: Adjunct. pp. 97-100. ACM (2016)

[47] King, R.e.a.: Body sensor networks for monitoring rowing technique. In: Body Sensor Networks (2009)

[48] Kohl, H.W.I., Cook, H.: Educating the student body: Taking physical activity and physical education to school. In: Committee on Physical Activity and Physical Education in the School Environment; Food and Nutrition Board, Institute of Medicine. pp. 199-199. National Academies Press (2013)

[49] Kong, K., Tomizuka, M.: A gait monitoring system based on air pressure sensors embedded in a shoe. IEEE/ASME Transactions on mechatronics 14(3), 358-370 (2009)

[50] Lau, A., Tong, R.: The reliability of using accelerometer and gyroscope for gait event identification on persons with dropped foot. In: Gait \& Posture, March (2008)

[51] Lichtenstein, M., T.T., J.: Exercise addiction in crossfit:prevalence and psychometric properties of the exercise addiction inventory. In: Addictive Behaviors Reports. pp. 33-37 (2016)

[52] Lika, B., Kolomvatsos, K., Hadjiefthymiade, S.: Facing the cold start problem in recommender systems. In: Expert Systems with Applications. pp. 2065 2073 (2014)

[53] Lin, F.e.a.: Automated patient handling activity recognition for at-risk caregivers using an unobtrusive wearable sensor. In: IEEE-EMBS International Conference on Biomedical and Health Informatics (BHI) (2016) 
[54] Lin, F.e.a.: Towards unobtrusive patient handling activity recognition for injury reduction among at-risk caregivers. In: IEEE Journal of Biomedical and Health Informatics (2016)

[55] Lin, F., Song, C., Xu, X., Cavuoto, L., Xu, W.: Sensing from the bottom: Smart insole enabled patient handling activity recognition through manifold learning. In: Connected Health: Applications, Systems and Engineering Technologies (CHASE). pp. 254-263. IEEE (2016)

[56] Lin, F., Wang, A., Zhuang, Y., Tomita, M.R., Xu, W.: Smart insole: A wearable sensor device for unobtrusive gait monitoring in daily life. IEEE Transactions on Industrial Informatics 12(6), 2281-2291 (2016)

[57] Lisboa, C.e.a.: A study for postural evaluation and movement analysis of individuals. In: XVIII Symposium on Virtual and Augmented Reality (2016)

[58] Manupibul, U., Charoensuk, W., Kaimuk, P.: Design and development of smart insole system for plantar pressure measurement in imbalance human body and heavy activities. In: Biomedical Engineering International Conference (BMEiCON), 2014 7th. pp. 1-5. IEEE (2014)

[59] Mariani, B.e.a.: On-shoe wearable sensors for gait and turning assessment of patients with parkinson's disease. In: IEEE Transactions on Biomedical Engineering, VOL. 60, NO. 1, January (2013)

[60] Martinez-Nova, A., Cuevas-Garcia, J.C., Pascual-Huerta, J., SanchezRodriguez, R.: Biofoot in-shoe system: Normal values and assessment of the reliability and repeatability. The Foot 17(4), 190 - 196 (2007). https://doi.org/https://doi.org/10.1016/j.foot.2007.04.002, http://www . sciencedirect.com/science/article/pii/S0958259207000338

[61] Matthies, D.J., Roumen, T., Kuijper, A., Urban, B.: Capsoles: who is walking on what kind of floor? In: Proceedings of 19th International Conference on Human-Computer Interaction with Mobile Devices and Services (2017)

[62] McCarthy, M., James, D., Lee, J., Rowlands, D.: Decision-tree-based human activity classification algorithm using single-channel foot-mounted gyroscope. Electronics Letters 51(9), 675-676 (2015)

[63] Michahelles, F.: Sensing and monitoring professional skiers. In: IEEE CS and IEEE ComSoc (2005)

[64] Moran S., Booker H., S.J., S., W.: Rates and risk factors of injury in crossfit: a prospective cohort study. In: Journal of Sports Medicine Physical Fitness. pp. 1147-53 (2017) 
[65] Morgan, P., Hansen, V.: Classroom teachers' perceptions of the impact of barriers to teaching physical education on the quality of physical education programs. In: Research Quarterly Exercise and Sport. pp. 506-516. National Institutes of Health (2008)

[66] Nguyen, U.e.a.: Increasing prevalence of knee pain and symptomatic knee osteoarthritis. In: Ann Intern Med. Dec 6; 155(11): 725-732 (2011)

[67] Noshadi, H., Dabiri, F., Ahmadian, S., Amini, N., Sarrafzadeh, M.: Hermes: mobile system for instability analysis and balance assessment. ACM Transactions on Embedded Computing Systems (TECS) 12(1s), 57 (2013)

[68] NXP: Mpl3115a2 datasheet. Https://www.nxp.com/docs/en/datasheet/MPL3115A2.pdf (2018), retrieved: June, 2018

[69] OECD: Obesity update. www.oecd.org/health/obesity-update.h (2017), retrieved: July, 2019

[70] Parkka, J.e.a.: Wearable static posturography solution using a novel pressure sensor sole. In: IEEE Transactions on Information Technology in Biomedicine, VOL. 10, NO. 1, January (2006)

[71] Particle.io: Electron datasheet. https://docs.particle.io/datasheets/cellular/electrondatasheet/ (2017), retrieved: July, 2019

[72] Perry, J., Burnfield, J.M.: Gait analysis: normal and pathological function. Developmental Medicine and Child Neurology 35, 1122-1122 (1993)

[73] de Pinho André, R., Diniz, P.H., Fuks, H.: Bottom-up investigation: Human activity recognition based on feet movement and posture information. In: iWOAR (2017)

[74] de Pinho André, R., Diniz, P.H., Fuks, H.: Investigating the relevance of sensor selection: Recognition of adls based on feet movement and posture information. In: Sensor Devices 2018 (2018)

[75] de Pinho André, R., Raposo, A.B., Fuks, H.: A platform for assessing physical education activity engagement. In: Intelligent Human Systems Integration. pp. 271-276 (2019)

[76] de Pinho André, R., Raposo, A.B., Fuks, H.: Using foot and knee movement and posture information to mitigate the probability of injuries in functional training. In: Human-Computer Interaction. pp. 153-169 (2019) 
[77] Rasberry, C., Lee, S., Robin, L., Laris, B., Russell, L., Coyle, K., Nihiser, A.: The association between school-based physical activity, including physical education, and academic performance: a systematic review of the literature. In: Preventive Medicine. pp. 10-20. Centers for Disease Control and Prevention (2011)

[78] Rippetoe, M.: Starting Strength. The Aasgaard Company (2011)

[79] Sazonov, E., Hegde, N., Browning, R.C., Melanson, E.L., Sazonova, N.A.: Posture and activity recognition and energy expenditure estimation in a wearable platform. IEEE journal of biomedical and health informatics 19(4), 1339-1346 (2015)

[80] Sazonov, E.S., Fulk, G., Hill, J., Schutz, Y., Browning, R.: Monitoring of posture allocations and activities by a shoe-based wearable sensor. IEEE Transactions on Biomedical Engineering 58(4), 983-990 (2011)

[81] Sazonova, N., Browning, R.C., Sazonov, E.: Accurate prediction of energy expenditure using a shoe-based activity monitor. Med Sci Sports Exerc 43(7), 1312-21 (2011)

[82] Sazonova, N.A., Browning, R., Sazonov, E.S.: Prediction of bodyweight and energy expenditure using point pressure and foot acceleration measurements. The open biomedical engineering journal 5, 110 (2011)

[83] Shu, L., Hua, T., Wang, Y., Li, Q., Feng, D.D., Tao, X.: In-shoe plantar pressure measurement and analysis system based on fabric pressure sensing array. IEEE Transactions on Information Technology in Biomedicine 14(3), 767-775 (2010)

[84] Simblee: Rfd77402 datasheet. https://www.digikey.com/en/datasheets/rfdigital-corporation/rf-digital-corporation-rfd77402 (2017), retrieved: July, 2019

[85] Smith M.M., Sommer A.J., S.B., S.T., D.: Crossfit-based high-intensity power training improves maximal aerobic fitness and body composition. In: The Journal of Strength \& Conditioning Research. pp. 3159-3172 (2013)

[86] Sollerhed, A.: Young today - adult tomorrow! Studies on physical status, physical activity, attitudes, and self-perception in children and adolescents. Ph.D. thesis, Lund University, Sweden (2006)

[87] Sparkfun: Battery pack datasheet. https://www.sparkfun.com/products/retired/14367 (2017), retrieved: July, 2019 
[88] ST: Lsm9ds1 datasheet. Https://www.st.com/resource/en/datasheet/Ism9ds1.pdf (2018), retrieved: June, 2018

[89] Tang, W., Sazonov, E.S.: Highly accurate recognition of human postures and activities through classification with rejection. IEEE Journal of Biomedical and Health Informatics 18(1), 309-315 (2014)

[90] Team, E.: Do not be fooled by the flexors. https://www.easyflexibility.com/blogs/yoga-flexibility-pearls/don-t-befooled-by-the-flexors (2017), retrieved: December, 2019

[91] Teixeira, M.e.a.: Clinical epidemiology of muscleskeletal pain. In: Revistas Médicas, São Paulo. Edição Especial. pp. 1-21 (2011)

[92] Terry, A., S.A., Griffiths, M.: The exercise addiction inventory: A new brief screening tool. In: Addiction in Research Theory. pp. 489-499 (2004)

[93] Tester, G., Ackland, T.R., Houghton, L.: A 30-year journey of monitoring fitness and skill outcomes in physical education: Lessons learned and a focus on the future. Published online at Scientific Research (7 2014)

[94] Ugulino, W., Cardador, D., Vega, K., Velloso, E., Milidiú, R., Fuks, H.: Wearable computing: Accelerometers' data classification of body postures and movements. In: Advances in Artificial Intelligence, SBIA, pp. 52-61. Springer (2012)

[95] Wahab, Y., Bakar, N.: Gait analysis measurement for sport application based on ultrasonic system. In: IEEE 15th International Symposium on Consumer Electronics (2011)

[96] Wallace, I.e.a.: Knee osteoarthritis has doubled in prevalence since the mid20th century. In: Proc Natl Acad Sci U S A. (2017)

[97] Weisenthal, B.e.a.: Injury rate and patterns among crossfit athletes. In: The Orthopaedic Journal of Sports Medicine (2014)

[98] Xue, Y., Jin, L.: A naturalistic 3d acceleration-based activity dataset \& benchmark evaluations. In: Systems Man and Cybernetics (SMC), 2010 IEEE International Conference on. pp. 4081-4085. IEEE (2010)

[99] Zhang, T., Fulk, G.D., Tang, W., Sazonov, E.S.: Using decision trees to measure activities in people with stroke. In: Engineering in Medicine and Biology Society (EMBC), 2013 35th Annual International Conference of the IEEE. pp. 6337-6340. IEEE (2013) 
[100] Zhang, Z., Poslad, S.: Improved use of foot force sensors and mobile phone gps for mobility activity recognition. IEEE Sensors Journal 14(12), 43404347 (2014)

[101] Zhu, C., Sheng, W.: Multi-sensor fusion for human daily activity recognition in robot-assisted living. In: International conference on Human robot interaction. pp. 303-304. ACM (2009) 